\title{
Earth Rotation Variations from Hours to Centuries
}

Jean O. Dickey

Jet Propulsion Laboratory, California Institute of Technology

Pasadena, California 91109-8099, USA

\begin{abstract}
The study of Earth rotation encompasses the complex nature of orientation changes, the excitation of these changes and their geophysical implications in a broad variety of areas. Studies have embarked on a new era with the advent of highly accurate space geodetic techniques and the increasing availability of complementary geophysical data sets. This paper provides an overview of Earth rotation variations from hours to centuries with a brief discussion of polar motion and nutation results.
\end{abstract}

\section{Introduction}

In the absence of internal sources of energy or interactions with astronomical objects, the Earth would move as a rigid body with its various parts (the crust, mantle, inner and outer cores, atmosphere and oceans) rotating together at a constant fixed rate. In reality, the world is considerably more complicated, as is schematically illustrated in Figure 1. The rotation rate of the Earth's crust is not constant, but exhibits complicated fluctuations in speed amounting to several parts in $10^{8}$ [corresponding to a variation of several milliseconds (ms) in the length of the day (LOD)] (Figure 2a) and about one part in $10^{6}$ in the orientation of the rotation axis relative to the solid Earth's axis of figure (polar motion) (Figure 3). These changes occur over a broad spectrum of time scales, ranging from hours to centuries and longer, reflecting the fact that they are produced by a wide variety of geophysical and astronomical processes. Geodetic observations of Earth rotation changes thus provide insights into the geophysical processes illustrated in Figure 1, which are often difficult to obtain by other means.

The principle of conservation of angular momentum requires that changes in the Earth's rotation must be manifestations of (a) torques acting on the solid Earth and (b) changes in the mass distribution within the solid Earth, which alters its inertia tensor. Angular momentum transfer occurs between the solid Earth and the fluid regions (the underlying liquid metallic core and the overlying hydrosphere and atmosphere) with which it is in contact; concomitant torques are due to hydrodynamic or magnetohydrodynamic stresses acting at the fluid/solid Earth interfaces. Changes in the inertia tensor of the solid Earth are caused not only by interfacial stresses and the gravitational attraction associated with astronomical objects and mass redistributions in the fluid regions of the Earth but also by processes that redistribute the material of the solid Earth, such as earthquakes, postglacial rebound, mantle convection, and movement of tectonic plates. Earth rotation provides a unique and truly global measure of natural and man-made changes in the atmosphere, oceans, and interior of the Earth.

Space geodetic techniques, including laser ranging to the moon and artificial satellites (LLR and SLR) and very long baseline interferometry (VLBI) using radio telescopes, have brought about a new age in Earth rotation and related studies. The length of day and 


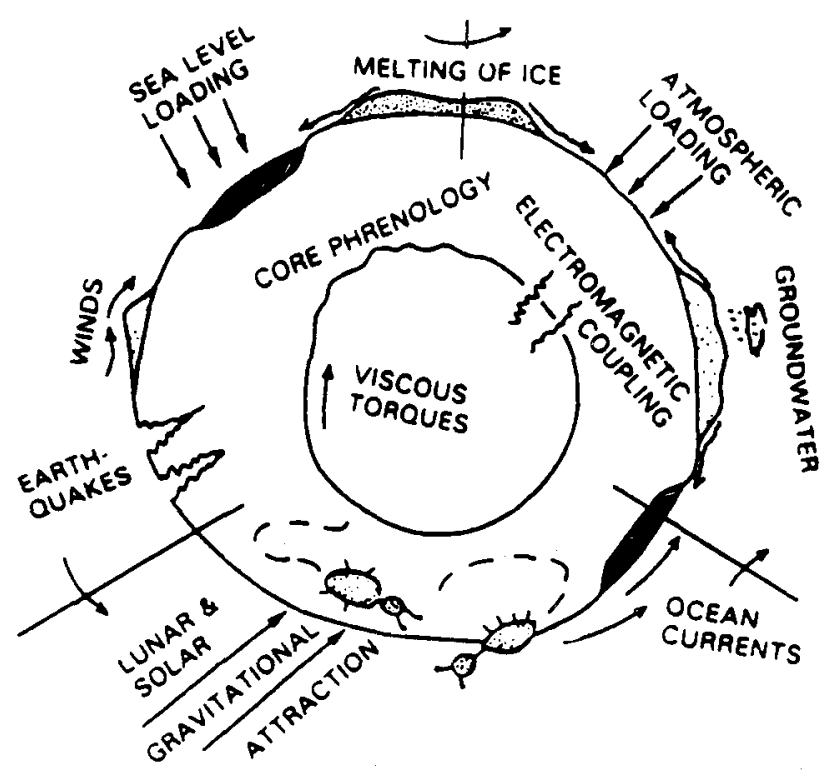

Fig. 1. Schematic illustration of the forces that perturb Earth rotation. Topographic coupling of the mantle to the core has been referred to as "Core Phrenology" by R. Hide, while the beetles, following T. Gold, represent the effect of continental drift. After Lambeck, $1980 \mathrm{~b}$.

polar motion are now routinely measured at the $\sim 0.3$ milliarcsecond level $(\sim 1 \mathrm{~cm})$ (Gross, 1994), while periodic corrections to the standard nutation model have been determined to the 0.1 milliarcsecond level for many terms (Herring et al., 1991). This paper provides an overview of Earth rotation variations from hours to centuries. Definitions and descriptions of the measurement techniques are given in Section 2, while a brief discussion of polar motion and nutations are given in Section 3 and 4. The main focus of this paper (Section 5 ) is on variations in universal time and length of day (LOD) and their implications. The reader is referred to several more detailed accounts of the excitation of Earth orientation changes. References to early work can be found in Munk and MacDonald (1960) and recent work is reported in Cazenave (1986); Dickey and Eubanks (1986); Eubanks (1993); Hide and Dickey (1991); Lambeck (1980 and 1988); Moritz and Mueller (1987); Rosen (1993); and Wahr (1983).

\section{Definitions and Measurements}

Earth rotation refers collectively to variations in the orientation of the solid Earth, encompassing Universal Time (UT1), length of day, polar motion, and the phenomena of precession and nutation. Universal time (UT1) is the rotation angle of the Earth: the direction of the Greenwich meridian in an equinox-based inertial frame. The UT1 measurements are usually given in units of time ( $1 \mathrm{msec}$ is $46 \mathrm{~cm}$ at the equator). The actual observable is UT1-T, where $\mathrm{T}$ is some independent time series such as ET (ephemeris time) or IAT (international atomic time). The LOD can be estimated from the time rate of change of UT1-T. The motion of the pole can be divided into two classes, nutational motion (which includes precession) and the polar motion (PM). Nutations are motions of the spin axis of the Earth with respect to an inertial reference frame, while 

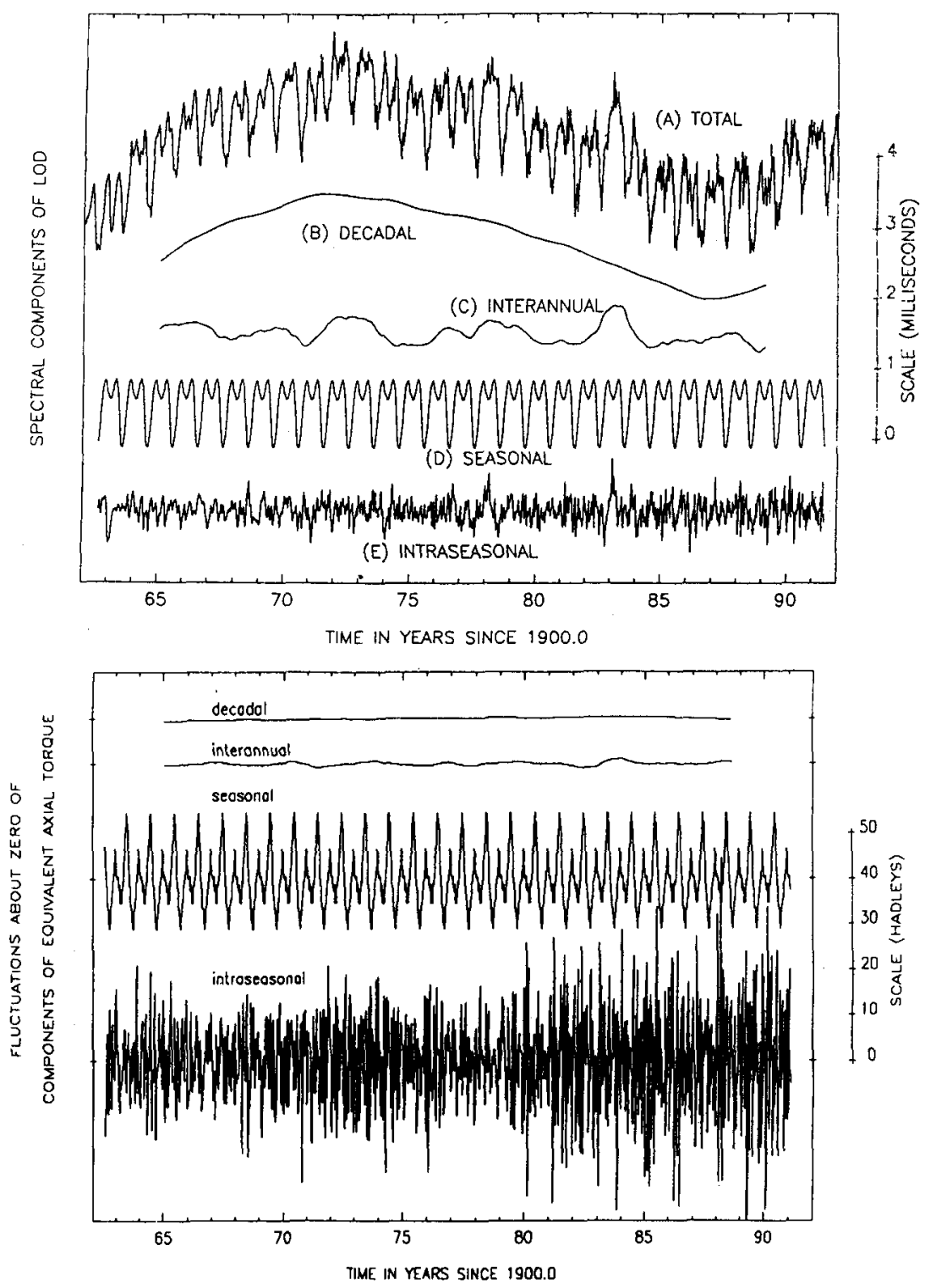

Fig. 2. Time series of irregular fluctuations in the length of the day from 1963 to 1992 (curve a) and its decadal, interannual, seasonal, and intraseasonal components (curves $b, c, d$ and $e$, respectively). The decadal (curve b) component largely reflects angular momentum exchange between the solid Earth and the underlying liquid metallic outer core produced by torques acting at the core-mantle boundary. The other components (curves $c, d$ and e) largely reflect angular momentum exchange between the atmosphere and the solid Earth, produced by torques acting directly on the solid Earth over continental regions of the Earth's surface and indirectly over oceanic regions. (b) Time series of the equivalent axial torques. Adapted from Hide and Dickey, 1991. 

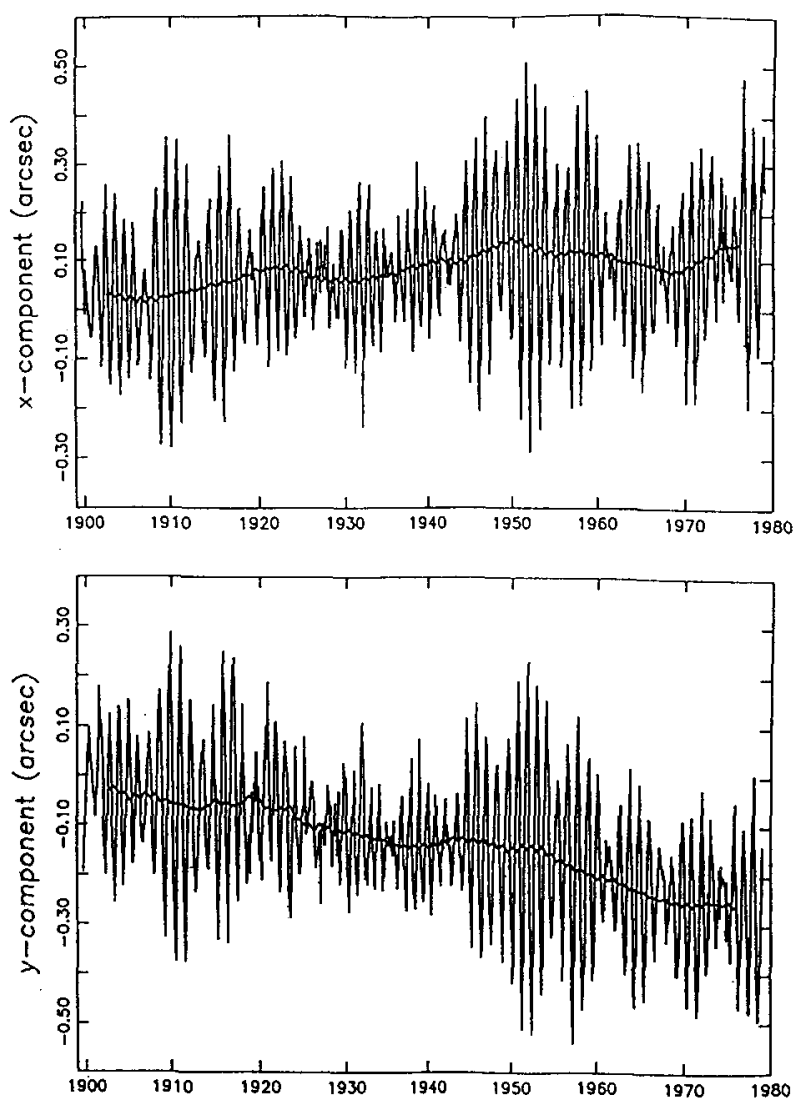

Fig. 3. Polar motion, 1900-1980, after Gross, 1990.

polar motion or wobble is the motion of the solid Earth with respect to the spin axis of the Earth. The precession is the gravest nutation with a period of about 26,000 years, caused by the gravitational torque on the Earth's equatorial bulge from the Sun, Moon and planets. Accurate knowledge of nutation and precession is required to relate terrestrial frames to celestial frames.

Earth orientation measurements in current use can be classified as arising from two sources: classical techniques (optical astrometry and lunar occultation) and modern space geodetic techniques. Optical astrometry is based on measurements of the apparent angular positions of selected bright stars (Feissel, 1980; Guinot, 1978). Both the latitude and the longitude of the observatory can be determined from observations of the time and elevation of the star at meridian passage. A network of stations with a proper geometry can be used to determine all components of the Earth's rotation. Polar motion has been routinely observed since 1900; from 1900 through 1980, the International Latitude Service (Yumi and Yokoyama, 1980) produced measurements based on a network of five stations located at the same latitude. Nutation values have been determined through the analysis of optical data sets beginning in 1955 (see for example Capitaine, 1980); the classical determination of the precession constant is based on the analysis of stellar proper motions (Fricke, 1977 and 1981). Prior to the advent of modern atomic clocks (1955), records of 
the solar time of ancient solar and lunar eclipses and of occultation of stars by the Moon form the basis for historical time series of UT1 and LOD (Stephenson and Morrison, 1984 and 1995).

The classical techniques were superseded by the new space geodetic techniques in the 1970s and 1980s. The new techniques are all based on measurements of electromagnetic signal delay or its time derivative. Delay and delay rate measurements are both more precise and somewhat less sensitive to systematic error than angular measurements. These measurement types include Very Long Baseline Interferometry (VLBI), satellite and lunar laser ranging (SLR and LLR), and utilization of the Global Positioning System (GPS) technology. VLBI is based on positional observations of distant quasars using the techniques of radio astronomy; SLR and LLR use range measurements to corner reflectors on artificial satellites and the Moon, respectively. GPS utilizes a large constellation of satellites transmitting at microwave frequencies together with a globally distributed network of receivers to determine universal time and polar motion variations. In each technique, changes in Earth orientation are monitored by observations of extraterrestrial objects from the surface of the Earth. The observed objects or signals are used to approximate a reference frame, either directly, for example, in the case of slow-moving objects such as quasars, or from dynamical theories of their motion in the case of planetary and satellite observations. In each case, Earth orientation is estimated from the apparent motion of the Earth with respect to this frame as defined by each technique. The accuracy of each technique is thus limited by unmodeled reference frame rotations, intrinsic measurement error, and any unmodeled motions of the observatories. In addition, measurements are most often referenced to some standard frame; hence, error is also introduced by the frame tie determinations.

Improvements in the observing systems (hardware, software and the development of well-distributed global networks) have lead to dramatic improvements in the determination of Earth orientation parameters. For example, estimates from a combined Kalman-filtered series utilizing the modern space techniques of LLR, SLR and VLBI have uncertainties in polar motion and universal time (UT1) at the $\sim 2$ mas $(6 \mathrm{~cm})$ and the $0.5 \mathrm{~ms}(\sim 23 \mathrm{~cm})$ level, respectively, for the late $1970 \mathrm{~s}$, whereas current uncertainties have improved to 0.3 mas for polar motion and $0.03 \mathrm{~ms}$ for UT1 (Gross, 1994). There has also been an impressive increase in time resolution. The classical optical technique, utilizing a global network of more than 50 observatories, obtained UT1 at the millisecond level at 5-day intervals (Feissel, 1980); in contrast, the SEARCH Campaign obtained sub-hourly measurements of both UT1 and polar motion (see Section V and Dickey, 1993).

\section{Polar Motion}

Polar motion is dominated by nearly circular oscillations at periods of one year (the annual wobble) and about 434 days (the Chandler wobble), with amplitudes of about 100 and 200 milliarcseconds (mas), respectively, together with a long-term drift of a few milliarcseconds per year (Lambeck, 1980 and Fig. 3). In addition, astrometric data sets exhibit decade time-scale polar motion of amplitude less than 50 mas, while analysis of geodetic data reveals rapid polar motion with peak-to-peak variations of approximately 2 to 20 mas, fluctuating on time scales between two weeks and several months; these are the so-called rapid polar motion (RPM) variations (Eubanks et al., 1988).

The equatorial momentum budget is not closed at any frequency; hence, research efforts are largely focused on uncovering the causes of the observed variations. Comparisons with meteorological data suggest that rapid polar motion variations are at 
least partially driven by surface air pressure changes, as modified by the response of sea level to atmospheric loading and by wind variations (Eubanks et al., 1988; Gross and Lindqwister, 1992). An additional source of excitation is required; a role for ocean angular momentum here has been advocated (Eubanks, 1993). The annual wobble is thought to be principally atmospheric in origin; however, detailed calculations (Chao and Au, 1991) indicate discrepancies with factor two disagreement between atmospheric and geodetic amplitude in the retrograde annual term and large phase discrepancy in prograde term, pointing to a need for an additional source of excitation.

The Chandler wobble is a free oscillation of the Earth (Smith and Dahlen, 1981; Wilson and Vincente, 1980) whose source of excitation is uncertain. Since polar motion is assumed to be a linear response to geophysical excitation, the problem is to find an excitation source with enough power near the Chandler period of about 434 days. The major candidates are the atmosphere and the redistribution of ground water. Atmospheric fluctuations have power in the Chandler frequency band and can excite $\sim 25 \%$ of the requisite power (Wahr, 1983). Other proposed sources, such as earthquakes, are probably too small in magnitude although great earthquakes may contribute (Gross, 1986). A complication in determining the amount of excitation needed is that we do not know what the dissipation of the mode (or $Q$ ) is. The current estimates of $Q$ assume a white noise excitation which may not be valid. If the $Q$ is increased sufficiently, the current excitation mechanisms may be enough to explain the Chandler wobble amplitude. Kuehne and Wilson (1991) investigated the effect of water storage in combination with air mass redistribution on the observed excitation of polar motion near the annual and Chandler frequencies for the period 1900-1985. They conclude that there is a discrepancy in accounting for more than half the variance of polar motion across a broad range of frequencies, suggesting a source of polar motion excitation due to air and water motion which has either not been correctly estimated or is not yet identified. Continual and improved monitoring of polar motion combined with improved models of atmospheric wind and pressure and ground water variations may resolve the degree to which the atmosphere and the hydrological cycle drive polar motion.

The changing distribution of water in the ground and oceans is likely to be important at periods of a year and longer. Decadal scale polar motion is dominated by motion that is linearly polarized along the direction that would result from sea-level rise or fall, suggesting that forcing is due at least in part to water mass redistribution (Wilson, 1993). On time scales of hundreds to thousands of years, water storage variation in the polar ice caps and the associated loading deformation of the solid Earth is a dominant influence on polar motion. Although one must account for other secular variations in these calculations, much of the observed secular drift of the pole in this century can be explained by redistributions of water and ice together with the post-glacial rebound following the last deglaciation around 20,000 years ago (Nakiboglu and Lambeck, 1980; Wu and Peltier, 1984). It is the zonally-symmetric part of the rebound that is responsible for the secular change in $J_{2}$ and other gravitational harmonics as observed by satellite laser ranging (see for example, Yoder et al., 1981 and Wahr et al., 1993 for a review). Polar motion data have been employed to calibrate and check viscoelastic rebound models that are used for studies of the Earth's interior and to predict relative changes in sea level. Contributions from plate motions, seismic deformation and present day melting of glaciers are also present. 


\section{Nutations and Precession}

Torques on the oblate Earth from the Sun, Moon and other bodies in the solar system cause a change in the direction of the Earth's rotation and figure axes, resulting in the precession of the Earth's equator along the ecliptic with superimposed oscillations. These motions are defined as the nutations. In addition to these forced nutations, there are free nutations which are internally caused. The response of the Earth to the externally applied torques is largely that of a rigid body. However, there are observable effects that arise because the Earth is deformable, with a solid-inner core, fluid-outer core, anelastic mantle, fluid oceans, and an atmosphere. It is these effects of the Earth's deformability that make nutations geophysically interesting (for a review, see Mathews and Shapiro, 1992). They serve as sensitive probes of Earth, allowing insights into the elasticity of the mantle, the effect of the oceans on the Earth's interior, coupling of the solid inner core to the fluid outer core and that of the fluid core to the mantle.

From an inertial coordinate system, the Earth's nutations have periods much longer than a day. The largest such motion is the long-period precession, a nutation of the spin axis with a period of about 26,000 years. The precession is caused by the gravitational torque on the Earth's equatorial bulge from the Sun, Moon and the planets and thus has an angular amplitude of $23.5^{\circ}$, the angle between the equator and the plane of the Earth's orbit (the ecliptic). The inclination of the Moon's orbit with respect to the Earth's equator induces a lunar nutation on the Earth. The Sun perturbs the lunar orbit and causes it to precess (rotate in the plane of the ecliptic) with a period of 18.6 years, with the induced nutation having the same period and an amplitude of about $9 \mathrm{~s}$ of arc. There are also nutations with periods of one solar year, one lunar month and at the harmonics of the dominant frequencies, with smaller nutations occurring at periods of half a solar year and a half-month and the harmonics of these. The half period is caused by the torque being symmetric about the equatorial plane.

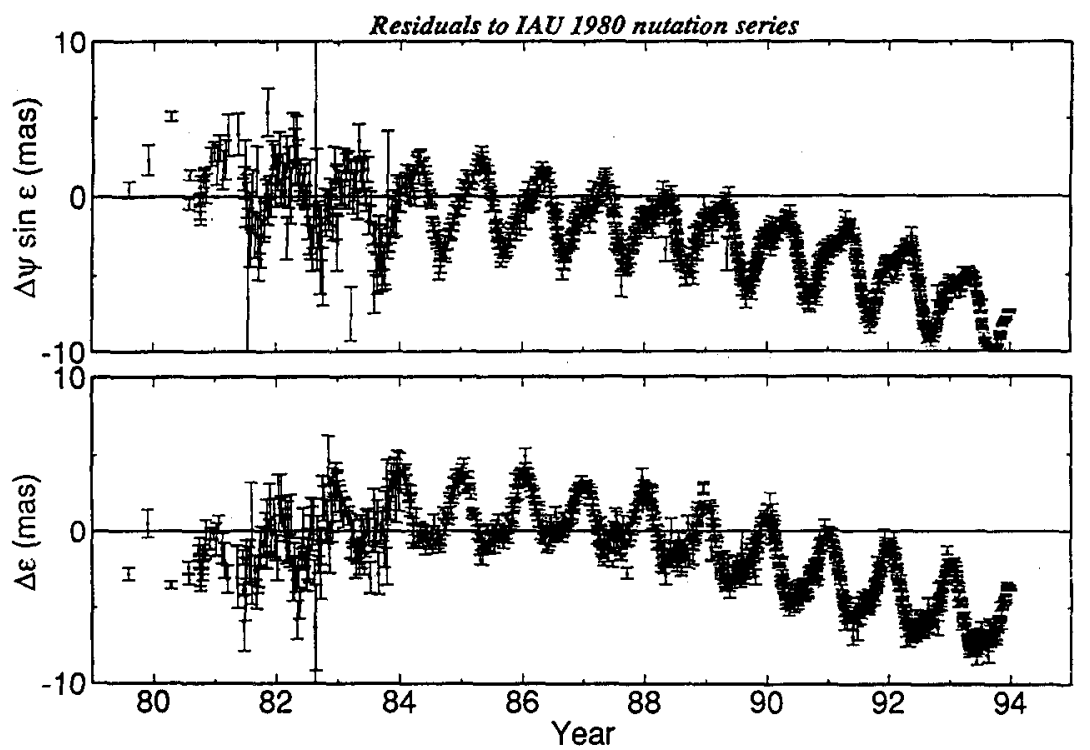

Fig. 4. Estimates of corrections, (a) $\delta \Delta \psi \sin \varepsilon_{o}$ and (b) $\delta \Delta \varepsilon$ to the IAU 1980 values for the nutation in longitude, $\Delta \psi \sin \varepsilon_{O}$, and the nutation in obliquity, $\Delta \varepsilon$ (4, T. Herring, Priv. Comm., 1993). 
Nutation series are calculated assuming that the individual periodic components can be linearly summed and that the motion of the body axis with respect to inertial space can be obtained from the addition of the periodic nutations, precession, and the motions due to polar motion. The currently recommended nutation model, the IAU 1980 Theory of Nutation (Seidelmann, 1982) is based on the work of Kinoshita (1977) for the rigid Earth series and of Wahr (1981) for the perturbing effects of elasticity on a rigid Earth model. Analyses of the data from the space techniques have indicated that sizable corrections are required to the standard nutation models, which are a consequence of the incompleteness of the geophysical models (see Figure 4$)$. The largest of these ( 2 mas) is the correction to the retrograde annual nutation, caused by the closeness to the free core nutation (FCN) response frequency (Herring et al., 1991), which has been interpreted as being due to the flattening of the core-mantle boundary which deviates from its hydrostatic equilibrium by about 5\% (Gwinn et al., 1986). A similar discrepancy between theory and observations has also been observed in tidal gravity data (Neuberg et al., 1987), with results which are consistent with this increased flattening of 5\%. Although the real parts of the FCN frequency agree well in both analyses, the tidal results have a significantly larger imaginary part (that is, smaller $Q$ than the geodetic results), the reason for which is under current investigation. The FCN source of excitation is an area of intense activity; one avenue that is being pursued is atmospheric excitation (see Joint Discussion on Nutation for further details).

\section{Universal Time (UT1) and Variations in the Length-of-Day}

Observed variations in the Earth's rate of rotation-or changes in the length-of-day (LOD)-are attributed to a variety of sources (Figure 1) and can be roughly divided into three categories: an overall linear increase mainly from tidal dissipation, irregular large variations on a time scale of decades, and the smaller higher frequency changesinterannual, seasonal and intraseasonal. The linear increase in the LOD, determined from ancient eclipse records (e.g., Stephenson and Morrison, 1984 and 1995) is estimated to be about 1 to $2 \mathrm{msec}$ per century. The LOD has large, irregular variations over time scales of decades to centuries (Figure 2a, curve B) with amplitudes of many milliseconds; these changes are too large to be of atmospheric origin and are thought to arise from fluid motions in the liquid outer core (Hide, 1977; Jault and LeMouël, 1991; Lambeck, 1980). Variations with periods of five years or less are driven primarily by exchanges of angular momentum with the atmosphere (Figure 2a, curves C, D and E). Estimates of the equivalent torques associated with each component, formed by taking centered differences in the time domain, are shown in Figure $2 \mathrm{~b}$. Although the amplitude of the decadal LOD time series is larger than that of the faster components (Figure 2a), the corresponding equivalent axial torque time series has the smallest amplitude (Figure 2b). At the other extreme, the most rapid intraseasonal contributions have the smallest amplitude, typically less than $0.2 \mathrm{~ms}$, but are associated with the largest torques. The decadal fluctuations integrate coherently over many years resulting in large variations; in contrast, the intraseasonal atmospheric excitations are quite sizable but cancel out to a large extent. The apparent increase in the magnitude of the intraseasonal torques during the period studied reflects improvements in both quality and quantity of Earth rotation data as well as better sampling methods, leading to a decreasing uncertainty in the LOD estimates with time. The Kalman filter used to produce this LOD series automatically adjusts the effective smoothing to the formal errors in the data, leading to increasing high-frequency variability in the latter part of the series, allowing more precise estimation of the magnitude of the 
shorter-period torques. These pronounced and rapid torque variations probably reflect changes in the orographic contribution to the total torque exerted by the atmosphere on the underlying planet, associated with the passage of synoptic weather systems over mountain ranges. Determinations incorporating space-geodetic data, in fact, show that the intraseasonal torque can change by as much as 50 Hadleys $\left(1\right.$ Hadley $=10^{18} \mathrm{Nm}$ ) in 10 days. Synoptic variations in the atmosphere occur on these time scales, associated with surface pressure gradients which, if applied across a mountain range with the dimensions of the Rockies or Andes, would produce transient torques of this magnitude. The seasonal torque, which, by definition, fluctuates with constant amplitude over the 30-year span of data used, reflects annual and semi-annual variations of the position and strength of the major atmospheric jet streams.

\section{Secular and Century-Time Scale Variations}

Irregular long-period variations in the rotation rate of the Earth were first hypothesized in the 19th century to account for otherwise inexplicable variations in the apparent motion of the Moon. This hypothesis was confirmed in the early part of the 20th century through observations of corresponding variations in the apparent motions of the planets. Variations in the Earth's rotation are not detectable unless the observations can be compared to a clock more stable than the Earth on the time scale of interest. This early work essentially used the orbital periods of the Moon and the planets as reference clocks; hence, the term Ephemeris Time (ET). The development of atomic frequency standards in 1955, stable to within a few parts in $10^{13}$ over indefinite periods marked a new era in "time keeping," and the time defined by these highly accurate clocks has become the standard (TAI). It is the variations $(\Delta T)$ of Universal Time (see Section 2$)$ from the uniform time scale that are of interest.

On century and long-term time scales, tidal dissipation, occurring both in the atmosphere and the oceans, is the dominant source of variability; the tidal bulge exerts a torque, causing the lunar orbit to expand and the Earth rotation rate to decrease. An analysis of twenty-five years of lunar laser ranging data provides a modern determination of the secular acceleration of the moon (n) of $-25.9 \pm 0.5$ arcsec/century ${ }^{2}$ (Dickey et al., 1994a) which is in agreement with satellite laser ranging results (see for example Marsh $e t$ al., 1990; Cheng $e t$ al., 1992). This implies a $\Delta t_{\text {TIDAL }}$ of $(42 \pm 2) t^{2}$ (note the units are seconds and $t$ is measured in centuries). Because of the quadratic term in $t$, historical data sets are best suited for a study of tidal dissipation. For example, $\Delta t_{\mathrm{TIDAL}}$ of $\sim 7$ hours have accumulated since 500 B.C.

A recent study by Stephenson and Morrison (1995) considers solar and lunar eclipses from the ancient and medieval civilizations of Babylon, Europe and the Arab world for the period 700 B.C. to A.D. 1600, lunar occultation data for the period A.D. 1600-1955.5, and modern data for the atomic clocks era (1955.5-1990). Both timed and untimed eclipse data were considered. Predictions based on modern tidal friction measurements $\left[42 t^{2}(\mathrm{sec})\right.$ or the corresponding LOD increase of $2.3 \pm 0.1 \mathrm{~ms} /$ century] does not fit the historical data; the best parabolic fit is $31 t^{2}$ (seconds) or a corresponding LOD increase of $1.70 \pm 0.05$ $\mathrm{ms} /$ century. The observed LOD increase can be interpreted as a composite of two actual trends: a tidal dissipation effect of $+2.3 \pm 0.1 \mathrm{~ms} / \mathrm{century}$ and non-tidal contribution of $-0.6 \pm 0.1 \mathrm{~ms} / \mathrm{century}$. The later effect is consistent with modern measurements of the change in the Earth oblateness $\left(J_{2}\right)$ (see Yoder et al., 1983 and Cheng et al., 1989) when the relaxation time of post-glacial rebound is considered. This is a quite remarkable result: the linking of 'space-based measurement from the last 20 years with ancient eclipse records 
of over 2500 years and the solving of a long-standing puzzle of relating current day $\dot{n}$ measurements to these ancient measurements.

\section{Decadal Variability}

Because we have no direct access to the core, Earth rotation observations are of prime importance in the study of the structure and dynamics of the Earth's deep interior. In general, torques can be produced at the core-mantle boundary by viscous, electromagnetic, topographic and gravitational coupling. Viscous coupling is likely to be comparatively very weak and would only be significant under extreme assumptions about the viscosity of the core. Two candidate mechanisms are currently under investigation for these decade variations: (1) topographic torques due to dynamic pressure forces associated with core motions acting on undulations of the core-mantle boundary and, (2) torques of electromagnetic origin. Difficulties, both of a qualitative and quantitative nature, are encountered when the electromagnetic coupling mechanism (first proposed by Bullard) is about (Paulus and Stix, 1986). Concerns include assumptions made concerning the strength of the toroidal part of the geomagnetic field in the outer reaches of the core and the distribution of electrical conductivity in the lower mantle. Estimates of topographic torques (first introduced by Hide, 1969) can be made from core motion deduced from geomagnetic secular variation, in combination with core-mantle boundary topography deduced either directly from seismic tomography or from dynamic models of the lower mantle based on tomographic and long-wavelength gravity anomaly data (Hide, 1989; Hide et al., 1993).

Geodetic torque estimates inferred from the LOD provide a means of checking results from seismology and geomagnetism and imposing constraints on the models used. Earth rotation results (Fig. 5) indicate a torque of $\sim 0.2$ Hadley units $\left(10^{18} \mathrm{Nm}\right)$ in the axial component during the 1980 period. When torque calculations are produced with the combined results from seismic tomography and geomagnetism for the 1970-80 decade, axial torque estimates are an order of magnitude too large; this model has "bumps" on the core-mantle interface being at the $3 \mathrm{~km}$ level. When a D" layer is included in the analysis (a boundary layer in the bottom 100-300 km of the mantle, which is though to have low viscosity), the undulations of the core-mantle boundary are $\sim 500 \mathrm{~m}$ (Hide et al., 1993) and the magnitude of the torque becomes consistent with LOD results. The size of these bumps is in accord with calculations based on corrections to the Standard IAU 1980 Nutation Model using Very Long Baseline Interferometry data (for example, Herring $e t$ $a l ., 1986)$. The corrections are dominated by a large retrograde annual term of $\sim 2$ mas. These observations can be interpreted as implying a core-mantle boundary that is more elliptical than for an Earth in static rotational equilibrium; the total required deviation from the figure of equilibrium is $500 \mathrm{~m}$ (Gwinn et al., 1986 and Section 4).

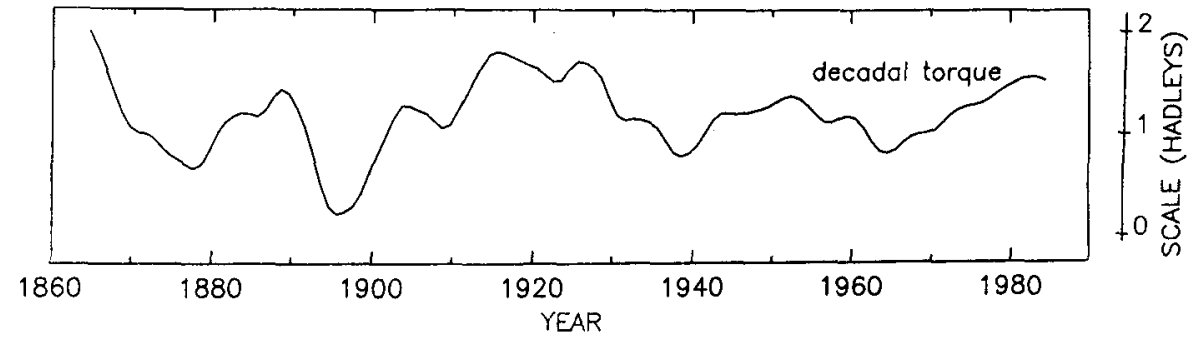

Fig. 5. Axial torque inferred from length-of-day data beginning in 1860 (see also Fig. 2). 
In a complementary approach to the torque calculation, one can examine the angular momentum exchange between the core and the mantle through the comparison of lengthof-day (that is, the mantle momentum) and core angular momentum (CAM). CAM is computed from time dependent core velocities derived from surface geomagnetic secular data (see for example, Jackson et al., 1993) using the results of the theory of Jault and LeMouël (1989) to extrapolate the zonal toroidal part of these motions throughout the core. It is the time-dependent component of this flow that transports angular momentum which can be compared with LOD (Fig. 6 and Jackson et al., 1989). A clear correlation is seen between the two series particularly after 1900; results indicate the core flow variations are of sufficient magnitude to explain decadal variations.

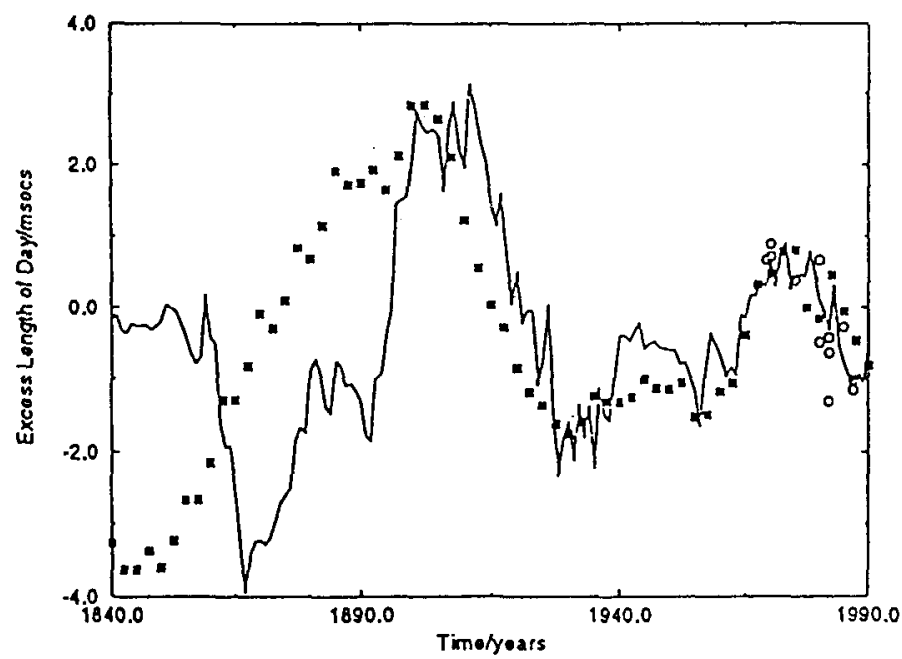

Fig. 6. Predicted excess length-of-day (over a standard day of 86,400 seconds) using the theory (Jault and LeMouël, 1989) for the period 1840-1990 (squares), compared to the observations (solid line) of McCarthy and Babcock (1986), corrected for secular trend with a value of $1.4 \mathrm{~ms}$ per century. Also shown are the results of Jault et al., 1988 (circles). After Jackson et al., 1993.

\section{Interannual Variability}

Several earlier studies have attempted to link interannual LOD variations with the stratospheric Quasi-Biennial Oscillation (QBO-characterized by the regular alternation of the zonal wind in the equatorial stratosphere at periods that vary from 24 to 30 months-for review, see Lambeck, Chapter 7, 1980). Initially, Lambeck and Cazenave (1977) associated interannual LOD changes during the 1955-71 period with the QBO. Later, Stephanick (1982) proposed a connection between interannual LOD and the "Southern Oscillation" by establishing coherence between LOD and equatorial Pacific air temperature (whose fluctuation is a good index of the Southern Oscillation phenomenon), speculating that the coherence was caused by zonal wind anomalies originating in the tropics. The coupled El Niño/Southern Oscillation (ENSO) phenomenon involves largescale redistribution of atmospheric mass between the eastern and western ends of the Pacific basin, and is associated with widespread changes in both atmospheric and oceanic circulation (Philander, 1990). Further investigations were stimulated by the occurrence of 
the unusually strong and well observed El Niño of 1982-83 (see e.g. Philander, 1983, 1990; Rasmusson and Wallace, 1983); the largest changes ever recorded in LOD and AAM occurred during this ENSO event (January and February, 1983) (Fig. 2-see also Rosen et al., 1984 and Eubanks et al., 1985). Various studies (Chao, 1984 and 1988; Eubanks et al., 1986; Dickey et al., 1990 and 1993) have established significant correlations between interannual LOD and the Southern Oscillation Index (SOI), computed as the seasonally-adjusted difference of the sea-level pressure between Tahiti and Darwin, Australia. During an ENSO event, the SOI reaches a minimum, and there is a concomitant collapse of the tropical easterlies and an increase in AAM. Conservation of total angular momentum requires the Earth's rate of rotation to diminish, causing LOD to increase. A further increase in AAM may result from the large-scale heating of the tropical troposphere associated with the El Niño events (Stephanick, 1982), leading to a zonallysymmetric rise in the tropical $200 \mathrm{mb}$ height field (Horel and Wallace, 1981) and a consequent strengthening of the upper-level subtropical jet streams.

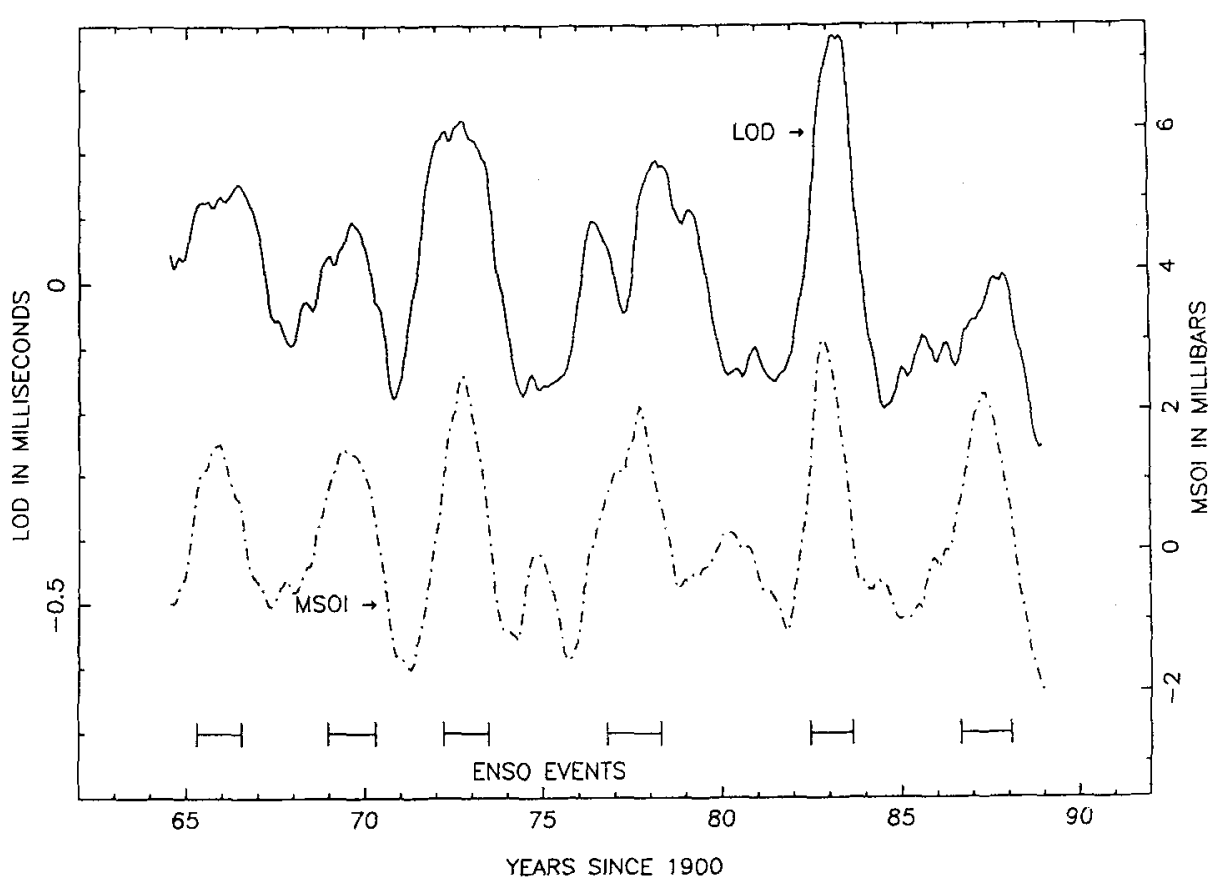

Fig. 7. The interannual LOD variation (computed as the 1-year moving average minus the 5-year moving average, top curve), compared to the negative of the interannual variation in the Modified Southern Oscillation index (MSOI, bottom curve).

The striking agreement between the interannual LOD variations and the MSOI [a modified Southern Oscillation index formed by taking the negative of the running annual SOI variation] is evident in Fig. 7 (Dickey et al., 1993a). Similar comparisons have been made with data sets beginning in 1860 to ascertain if LOD can be used as a proxy index of interannual global wind fluctuations (Salstein and Rosen, 1986; Dickey et al., 1990 and 
1993; Jordi et al., 1994). Results indicate correlations are robust enough that LOD can be used as a proxy index beginning in about 1930 (Dickey et al., 1993; Jordi et al., 1994). The maximum cross-correlation in the modern data $(0.72)$ is found with the MSOI leading LOD variations (and the transfer of angular momentum to the solid Earth) by about 2 months. Atmospheric winds play the dominant role in these variations, accounting for up to $92 \%$ of the LOD variation in a case study of the 1982-83 El Niño (Dickey et al., 1994). The stratosphere is an important contributor, as it accounts for as much as an additional $20 \%$ in the LOD variance explained relative to the winds below $100 \mathrm{mb}$. The LOD variance not accounted for by AAM during the case study ( $55 \mu \mathrm{s}$ rms) is larger than the difference between interannual variations in data from the $\mathrm{NMC}$ and $\mathrm{EC}$ (18 $\mu \mathrm{s} \mathrm{rms}$ ), indicating that "noise" in the AAM data may explain a part, but not all, of the residual. Additional sources of discrepancy include systematic AAM error (estimated to be $\sim 30 \mu \mathrm{s}$ ) and a possible oceanic contribution (Dickey et al., 1994c).

Additional insight into the origin of interannual rotational fluctuations can be gained through the examination of the latitudinal structure of the associated atmospheric variation (Dickey et al., 1992a). The AAM obtained by integrating atmospheric data up to $100 \mathrm{mb}$ over 46 equal-area belts is considered, with interannual variation obtained by differencing the one-year and five-year moving averages for each latitude band. The resulting pattern of interannual variability (Fig. 8) is striking, with coherent V-like structures emerging from the tropics and propagating poleward. The 1982-1983 event is associated with positive AAM (that is anomalous eastward winds) beginning at the equator in late 1980/early 1981 and propagating to high latitudes in both hemispheres over the course of several years.

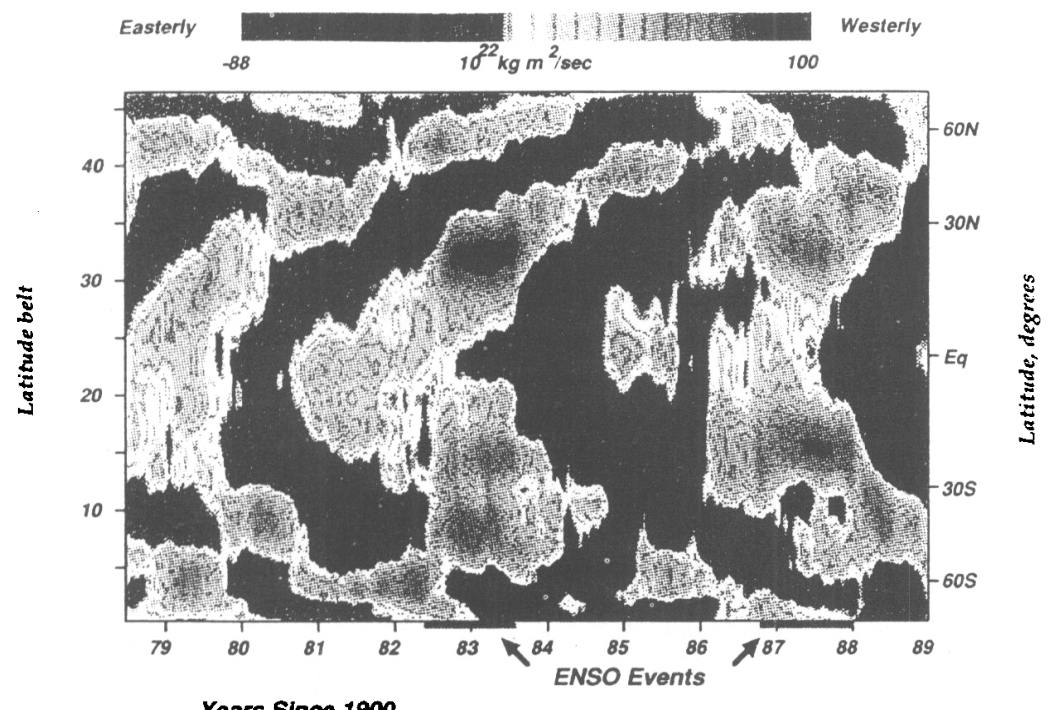

Fig. 8. Latitude-time (Hovmöller) plot of interannual atmospheric angular momentum variations from the National Meteorological Center analysis over 46 separate equal area latitude bands, based on atmospheric wind data integrated up to $100 \mathrm{mb}$. The interannual variations shown were obtained by differencing the one-year and five-year moving averages for each individual band (after Dickey $\boldsymbol{t} t$ al., 1992a). 
During the mature phase of the ENSO event (mid-1982 to mid-1983), strong positive anomalies are located in the northern subtropics with moderate anomalies in the southern subtropics. These anomalies diminish in strength and propagate poleward as the ENSO event decays. A similar scenario can be seen for the 1986-87 event, with strong modulation on the QB time scale (Dickey et al., 1992a). These low-frequency variations may represent a combined mode of the coupled global ocean-atmosphere system, whose precise origin is under investigation (Marcus and Dickey, 1994).

\section{Seasonal and Subseasonal Variations}

The exchange of angular momentum between the atmosphere and solid Earth is evident in fluctuations in the length-of-day at periods of a year and less (Fig. 9). Recent improvements in Earth rotation measurements have been accompanied by improvements in numerical models and measurements of Earth's global atmosphere which can be used to calculate the atmospheric angular momentum (AAM) (see for example, Salstein et al., 1993). Both U.S. and foreign meteorological services maintain global atmospheric models for weather forecasting; surface and upper-air wind data and other meteorological measurements are assimilated into these models on a regular basis. Certain atmospheric variables, including pressure and horizontal wind velocity, are estimated at each model grid point at twelve-hour intervals by combining measurements of these variables with their forecasted values in a statistically optimal fashion. Calculation of an effective atmospheric excitation function, $\chi$, a three-dimensional pseudo-vector including Love number corrections for rotational and surface loading deformation of the Earth, can be made directly from the meteorological data (Barnes et al., 1983).

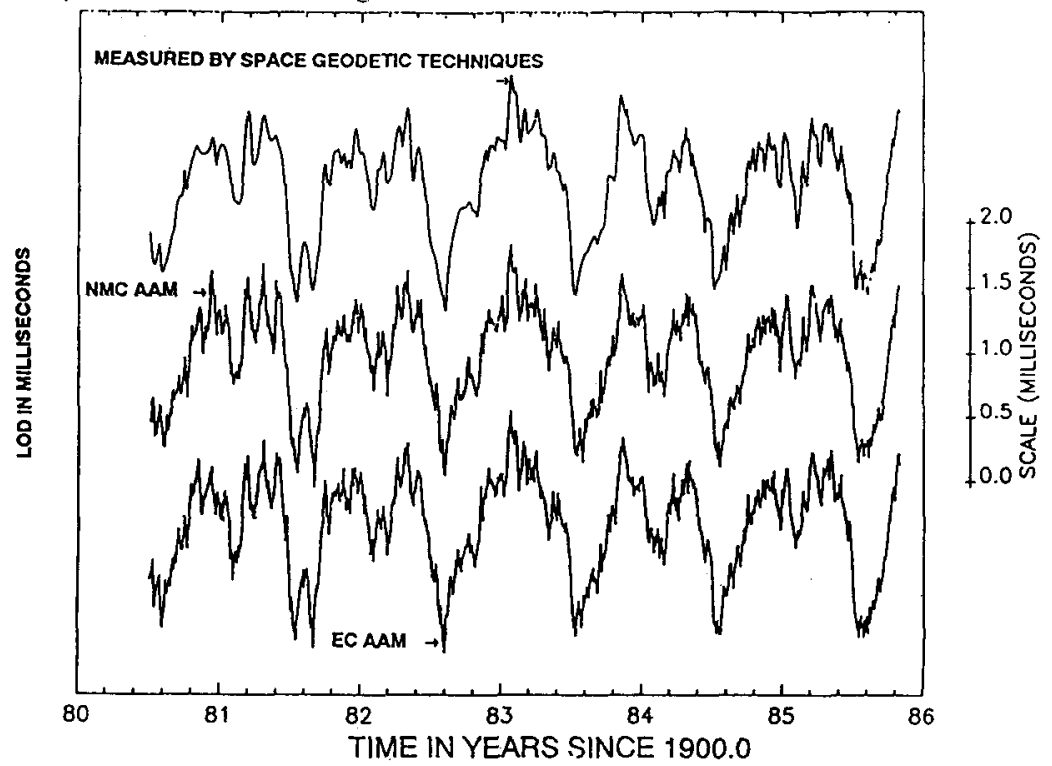

Fig. 9. Time series of the sum of the seasonal and intraseasonal LOD components, with the upper curve measured by space geodetic techniques (see Fig. 2a) and the middle and bottom curves inferred from routine daily determinations of changes in the axial component of the atmospheric angular momentum made by the U. S. National Meteorological Center and the European Centre for Medium Range Forecasts, respectively (after Dickey et al, 1993b). 
Comparison of astronomical measurements with axial atmospheric angular momentum first indicated the significance of the atmospheric contribution to Earth rotation. Various studies (for a review see Hide and Dickey, 1991; Dickey, 1993; Eubanks, 1993 and Rosen, 1993) have related LOD fluctuations to changes in atmospheric angular momentum on time scales ranging from months to a few years using mean monthly or longer period atmospheric data. Hide et al. (1980), comparing the wind component of angular momentum evaluated at 12-h intervals from the First GARP Global Experiment (FGGE) with LOD data, demonstrated that angular momentum transfer between the solid Earth and the atmosphere could fully account for the observed LOD variation on time scales of days to weeks. Intercomparisons of AAM data with modern LOD data obtained from space geodetic techniques (see for example, Fig. 9) have been at the forefront of recent research on seasonal and sub-seasonal fluctuations in the Earth's angular momentum budget. The correlation between short-period variations in length-of-day and AAM is so well established that numerical forecasts of atmospheric angular momentum are now being used as a proxy data type for the purpose of predicting Earth rotation variations (Freedman et al., 1994a).

Changes in LOD at seasonal and higher frequencies can be attributed primarily to exchange of angular momentum with the atmosphere, with zonal frictional stress and surface pressure gradients acting as agents for transferring angular momentum between the atmosphere and the solid Earth. Changes in both the AAM and LOD contain a large seasonal cycle, dominated by annual and semiannual harmonics (Fig. 9). The seasonal cycle of angular momentum causes seasonal LOD changes with an amplitude of about 0.5 msec, and UT1 changes with an amplitude of about $30 \mathrm{msec}$. The annual angular momentum cycles in the two Hemispheres are nearly 180 degrees out of phase, with the Northern Hemisphere having a larger amplitude. The annual cycle is mostly due to changes in the mid-latitude westerly (i.e., eastward propagating) winds, particularly the subtropical jet streams at or near the 250 millibar level (Rosen and Salstein, 1983). Various studies of the small apparent discrepancies found in AAM-LOD intercomparisons (Eubanks et al., 1985a; Morgan et al., 1985) reveal that imbalances occur in the angular momentum budget at the semi-annual period and to a lesser extent at the annuel period. The seasonal imbalances can be attributed to the failure to represent contributions from stratospheric winds (up to the $1 \mathrm{mb}$ level) satisfactorily in the routine operational analyses (Rosen and Salstein, 1985 and 1991). Currently, closure of the angular momentum budget is within the current measurement errors, with the ocean contribution being comparable in size (Dickey et al., 1993 and Ponte and Rosen, 1994). Further refinements will require the full consideration of the global oceans.

Superimposed on the annual cycle in both geodetic and atmospheric data is the irregular "intraseasonal" fluctuation, evident on time scales ranging from roughly 40 to 50 days (Fig. 9). The corresponding oscillations in zonal winds and other meteorological quantities were first discovered by Madden and Julian $(1971,1972)$ in station data from the equatorial Pacific. Since that time, higher-frequency oscillations have also been seen (Miller, 1974) and the 40 to 50 day oscillation has been observed on a global scale (Krishnamurti and Subrahmanyam, 1982; Yasunnari, 1981; Anderson and Rosen, 1983). The corresponding Earth rotation changes were first detected in optical data (Zheng, 1979) and confirmed by their presence in four independent UT1 data types (Feissel and Gambis, 1980); Langley et al. (1981) later reported this effect in both the AAM and LOD from lunar laser ranging. Feissel and Nitschelm (1985) showed the intermittent variability of this oscillation. The peak amplitude seems to be about $0.2 \mathrm{msec}$ of length of day, though the oscillation exhibits changes in both period and amplitude on a year-to-year basis. 
Morgan et al. (1985) showed that any nonmeteorological contribution to the 40-50 day oscillation is not significantly larger than the uncertainties in the observations (about 0.06 $\mathrm{ms}$ ).

Three possible mechanisms for the origin have been proposed. The so-called "MaddenJulian" mechanism connects these intraseasonal LOD and AAM oscillations to eastwardpropagating anomalies in tropical convection and zonal wind (Madden, 1986 and 1987). A second hypothesis (Ghil, 1987; Ghil and Childress, 1987) relates the oscillation to an instability of the nonzonal westerly flow caused by the interaction of the jet stream with mountains in the mid-latitudes. The dominant period of this instability in a simplified, equivalent-barotropic model of the atmosphere is near 40 days; however, for realistic parameter values, it has aperiodic, intermittent behavior, which would explain the broadband nature of the AAM/LOD oscillations. The third mechanism, proposed by Simmons $e t$ al. (1983), links these oscillations to atmospheric disturbances which derive their energy from the basic state through barotropic instability. In this theory, topography contributes only to the maintenance of asymmetries in the climatological basic state, and is absent from the instability mechanism itself; in the second approach, topography interacts both with the basic flow and with its oscillatory instability.

The analysis of Earth rotation variations in concert with AAM data provides novel insights into the intraseasonal oscillation and other geophysical processes. Studies of the spectral characteristics of signal-to-noise ratios in both data types, for example, indicate that LOD determinations give a more accurate picture of 40 to 50 day variations in the global angular momentum budget than can be obtained at present from the available AAM determinations (Dickey et al., 1992b). By compositing Earth rotation data over several years, the global intraseasonal oscillation was found to be strongest during Northern Hemisphere winter (Dickey et al., 1991). These data indicate that the low-frequency variability of the Northern Hemisphere extratropics may be important to the dynamics of the global oscillation, as envisaged in the second and third hypotheses (Dickey et al., 1991; Marcus, 1990; Marcus et al., 1990). Spectral analysis of modern LOD data shows the presence of two distinct intraseasonal oscillations, with periods near 50 and 40 days respectirely. Studies of concurrent AAM variations from the National Meteorological Center (NMC) operational analysis, and from perpetual-January runs with the UCLA General Circulation Model, indicate that the larger-amplitude 50-day oscillation arises in the tropics, whereas the 40-day oscillation originates in the Northern Hemisphere extratropics. Thus, comparisons of geodetically-determined LOD values with both observed and simulated AAM data indicate that the global intraseasonal oscillation may result from a combination of all three aforementioned mechanisms. Several issues remain open, including whether these oscillations are independent and what feedback mechanisms operate between these two oscillation.

\section{High Frequency Variations}

High time resolution measurements of Earth rotation and atmospheric angular momentum (AAM) and torque and their interpretation are improving our understanding of the properties and origin of short-period fluctuations of the Earth's orientation, and are providing new and unique insights into a variety of geophysical processes. These processes include dynamical coupling and angular momentum exchange between the solid Earth and atmosphere, the interaction of ocean tides with the Earth's rotational dynamics and the effects of fluid-core resonances. The importance of the determination of rapid Earth rotation variations and its implication for geodynamics was recognized by the 
International Union of Geodesy and Geophysics (IUGG) in Vienna (August 1991) through a Union resolution and by the formation of a joint IUGG/IAU Working Group, Rapid Earth Orientation Changes. As a result, a major campaign, SEARCH'92 (Study of EarthAtmosphere Rapid CHanges), for high time resolution (sub-daily) measurements of Earth rotation by all of the space geodetic techniques was coordinated by the International Earth Rotation Service (IERS) and was held in conjunction with the International GPS Service (IGS) Campaign (June 21-September 22, 1992). A special intensive period (Epoch'92) extended from July 25 through August 10, 1992 (Dickey and Feissel, 1994).

GPS measurements were obtained from a core network of $\sim 30$ receivers with 80 additional sites in place during EPOCH'92 (Beutler and Brockman, 1993). The SLR global network consisted of 30 sites, two of which are dual SLR/LLR stations and obtained LLR results as well. The VLBI measurement program consisted both of routine operations (IRIS-A, NAVNET) as well as intensive observing efforts. There was essentially continuous coverage on two simultaneous VLBI networks during the period July 27-August 10 (for an overview, see Dickey et al., 1994c). Special efforts were made to obtain the best possible auxiliary data from geophysical, oceanographic, and atmospheric sources. The frequency of archived atmospheric angular momentum calculation was increased from twice to four times daily and atmospheric torque calculations were begun.

An additional campaign, CONT'94, was held in January 1994, which allows for a study of solid Earth-atmosphere interaction during the Northern Hemisphere winter. This activity featured 15 days of continuous VLBI with 21 stations in three simultaneous networks coupled with participation by the other techniques. The result was the best VLBI to date: $6 \mathrm{~mm}$ repeatability on $10,000 \mathrm{~km}$ baselines, and highly accurate hourly measurements of Earth rotation ( $6 \mu$ s in UT1 and $250 \mu$ as in PM).

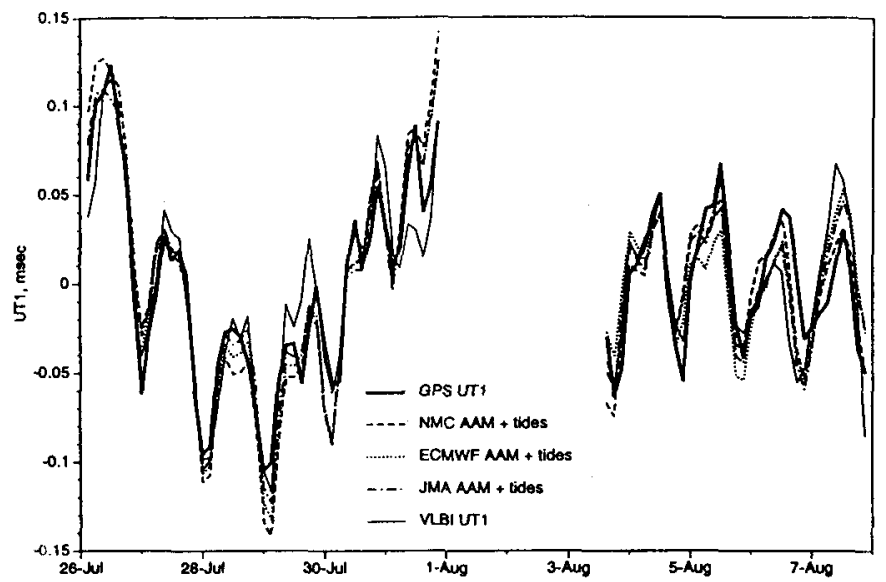

Fig. 10. The sum of the integrated atmospheric angular momentum (AAM) and diurnal, semidiurnal, and longer period tides compared with the observed UT1 variations from GPS and VLBI. Three AAM series are shown: U. S. National Meteorological Center (NMC), European Centre for Medium Range Forecasts (ECMWF), and Japanese Meteorological Agency (JMA). After (Freedman et al., 1994b). 
A strong diurnal and semidiurnal signature is clearly seen in all data types (see Fig. 10); comparisons with the independent techniques of VLBI and GPS confirm the reality of this signature $[\sim 0.1 \mathrm{msec}(5 \mathrm{~cm})$ in amplitude]. The data gap in the middle of the time series is due to the use of anti-spoofing (AS) signal encryption in the GPS. Diurnal and semi-diurnal rotational variations were postulated by Yoder et al. (1981), who proposed that such signatures should arise from the interactions of the ocean tides with the solid Earth. Estimates of these variations were made by Baader $e$ t al. (1983) for the $\mathrm{M}_{2}$ tide and were refined by Brosche et al. (1989 and 1991) for the major diurnal and semidiurnal tides. Seiler (1991) and Wünsch and Seiler (1992) revised the Brosch et al. (1989) tidal model using a new numerical ocean model; these calculations were later improved by Gross (1993) through the use of a more realistic rotation model (see also Brosche and Wünsch, 1994). Dickman (1993) developed the "broad-band" Liouville equation approach and determined the effects of the dynamic ocean tides on Earth rotation. The models mentioned thus far are unconstrained in that the solutions are not required to fit in situ tidal measurements and depend only on knowledge of the global bathymetry. Such an approach requires sophisticated hydrodynamic modeling with fine spatial resolutions in shelf areas, as the tidal energy dissipation occurs mainly in the shallow seas.

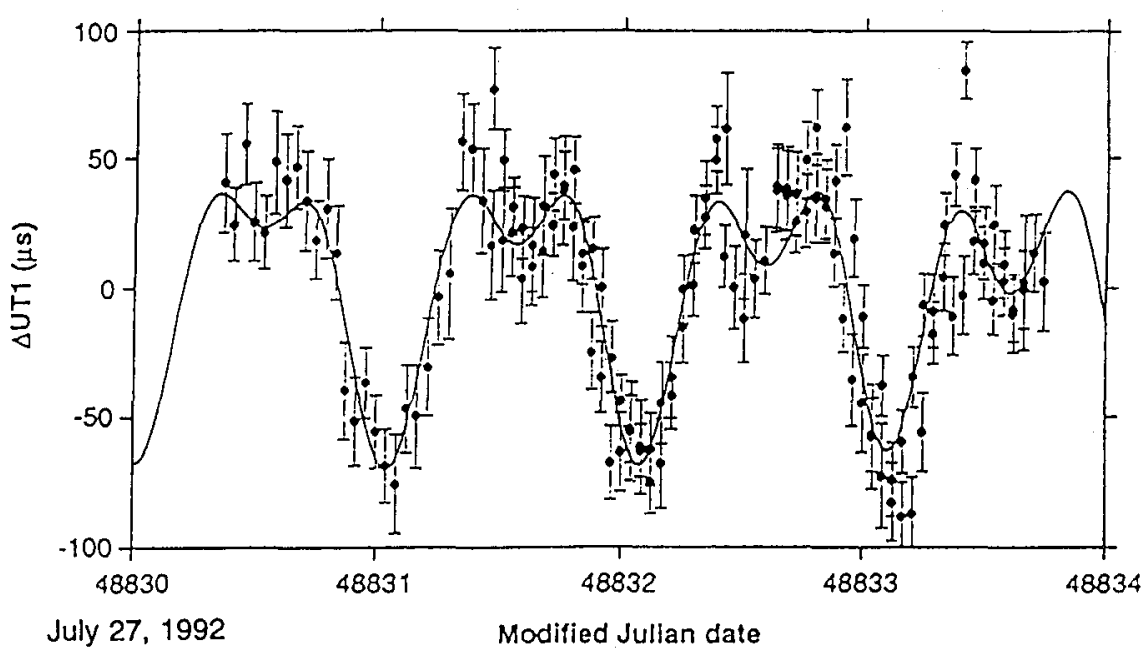

Fig. 11. Predicted and observed variations in $\Delta U T 1$. The predictions (solid curve) are based on a tidal-height model. The observations with $1 \sigma$ standard errors, are the product of five overlapping VLBI experiments carried out during late July 1992 (modified Julian date $48830=27$ July 1992). Each experiment was about 1 day long, and each has been adjusted here empirically by a single bias. After Ray et al., 1994.

A class of global tide models have been developed that are constrained by tide observations (gauge measurements at coastlines and at islands, and satellite altimetry). 
This alleviates to a certain degree the need for high spatial resolution models with complex physics packages. In a recent study, Ray et al. (1994) utilized the model of global tidal heights derived by Schwiderski (1980), which is constrained by more than 2000 coastal, island and bottom pressure measurements. In addition to the effect of the global height fields, the impact of the tidal currents inferred from a modified form of Laplace's momentum equation were included. A complementary approach is the development of empirical models obtained by fitting the major tidal components to sub-daily Earth rotation observations. This approach has been applied to VLBI analysis by Sovers et al. (1993) and Herring and Dong (1994) and to SLR analysis by Watkins et al. (1994). As one would expect, the empirical and constrained models result in better fits than the unconstrained. Differencing with respect to the Brosche et al. (1989) model as modified by Gross (1993) actually increases the scatter. Most of the discrepancies arise from the semidiurnal band, which the theoretical model predicts to have a much larger amplitude (Freedman et al., 1994b). Ray et al. produce tidal variations in Universal Time that agree with VLBI observations at a 2 microsecond level (see Fig. 11) and thus establish oceanic tides as the dominant mechanism for producing Earth rotation variations at these periods.

Moving to multiple day time scales, one can add the atmospheric effect to the tidal variations (here the empirical model of Herring and Dong (1994) is used) by integrating AAM to obtain the atmospheric variability (Fig. 10). Linear trends were removed from each series for each of the two periods. The geodetic signal can be described by the sum of AAM variation and tidally induced UT1, with tides acting at periods of one day and less and AAM dominating variations at periods greater than a day. The differences between GPS and VLBI are at least as large as those between the AAM series themselves and the AAM and geodetic series. There is no residual signal that exceeds the formal errors. The subdaily variability of the AAM is quite small and at this point cannot be separated from the oceanic effects; however, limits can be expressed (Freedman et al., 1994). Improved theoretical tide models are need to unravel the oceanic and atmospheric signals, with geodetic measurements providing strong constraints. The creation of a new generation of ocean models is now underway through the analysis of TOPEX (Ocean Topography Experiment)/Poseidon satellite altimeter mission data. These developing models, particularly those that utilize data assimilation, should provide greatly improved predictions of tidally induced Earth rotation variations (Ray et al., 1994)

During a 6-day subperiod (July 31-August 5, 1992) within the SEARCH Campaign, a strong rise in both LOD and AAM was observed, which was caused primarily by zonal wind variations. Analysis of equal area belts of AAM data indicates that the low-latitude Southern Hemisphere belts are the dominant contributors to these effects (Salstein and Rosen, 1994). While angular momentum variations provide information on the center of activities during the period of interest, additional insight into the mechanisms involved can be gained through the analysis of the atmospheric torques. In the study of Salstein and Rosen (1994), both mountain torque (which results from differences in normal pressure forces across mountain barriers) and friction torque (which arises from tangential frictional stresses at the atmosphere's lower boundary) are considered. Mountain torques accounted for most of the momentum transfer between the solid Earth and atmosphere, with the Southern tropics $\left(0^{\circ}-30^{\circ} \mathrm{S}\right)$ making the largest contribution. Note that this campaign was held in Southern Hemisphere Winter; hence, the bulk of the activities are expected there. Torques across South America are particularly important. This event is associated with a high-pressure system east of the Andes Mountains that produced a strong zonal pressure gradient, thus inducing the observed AAM and LOD variations. 


\section{Concluding Remarks}

Earth orientation studies provides a unique and truly global measure of natural and manmade changes in the atmosphere, oceans, and interior of the Earth, and insights into the interactions of the Earth, Moon and Sun. They require the combined efforts of many disciplines, enforce a global perspective and promote mutual interaction. They are concerned with processes lasting from minutes to millions of years, relating phenomena occurring over vastly different time scales (such as earthquakes and tectonic motion; seasonal water storage and glacier dynamics, and weather and long-term climate change). Because of their very nature, the measurement and analysis programs promote international cooperation.

The new space geodetic techniques have had a profound influence on Earth orientation studies. Typical uncertainties resulting from conventional optical observations were at the $1 \mathrm{~ms}$ level for Earth rotation and 10 mas for polar motion. Today's uncertainties have decreased dramatically by more than an order of magnitude, in some cases approaching two orders. The length-of-day and polar motion are now routinely measured at the $\sim 0.3$ millarcsecond level $(\sim 1 \mathrm{~cm})$ (Gross, 1994), while periodic corrections to the standard nutation model have been determined to better than the 0.1 mas level for many terms (see Joint Discussion on Nutations, this volume). In addition, the time resolution has been increased significantly. Prior to 1980, the BIH optical results were averaged from a global network of stations with a time resolution of 5 days; in contrast, results from CONT'94 produced multihour solutions with accuracies for UT1 at the $6 \mu \mathrm{sec}$ level and at the 240 $\mu$ as level for polar motion.

These advances have been accompanied by improvements in a number of related areas. Prime examples include atmospheric measurements and numerical models of the Earth's global atmosphere, which are used to calculate the atmospheric angular momentum and other synergistic atmospheric data sets. Much insight is gained through joint analysis of geodetic observations with complementary geophysical data including results from seismology, geomagnetic models and general circulation models (GCMs) of both the atmosphere and the oceans.

The effects of the Earth's various subsystems are evident over a wide range of frequencies. Subdaily variations are largely excited by oceanic tides, while on time scales from days to interannual, atmospheric excitation dominates. Core-mantle coupling is the leading candidate for the excitation mechanism on decadal time scales, whereas tidal dissipation and the effect of post-glacial rebound are clearly seen on century time scales.

Considerable progress has been made; however, many open issues remain. What is the role of the oceans on interannual and seasonal time scales? What is the atmospheric contribution to long-term variation? Thus far, we have largely focused on the angular momentum exchange approach; with the advent of improved measurements and models, further insights can be gained through the torque approach. Many fundamental issues remain unresolved in polar motion studies, as it is difficult to account for the full polar motion excitation source across a wide frequency band. The outlook for progress in these areas is bright, and with it the promise of gaining a unifying perspective of planet Earth.

Acknowledgments. The author gratefully acknowledges S. L. Marcus for helpful comments on this manuscript, and T. A. Herring for Figure 6. The work of the author presents the results of one phase of research carried out at the Jet Propulsion Laboratory, California Institute of Technology, sponsored by the National Aeronautics and Space Administration. 


\section{REFERENCES}

Anderson, J. D., and R. D. Rosen, The Latitude-Height Structure of 40-50 Day Variations in Atmospheric Angular Momentum, J. Atmos, Sci., 40, 1584-1591, 1983.

Baader, H.-R., P. Brosche, and W. Hovel, Ocean Tides and Periodic Variations of the Earth's Rotation, J. Geophys., 52, 140-142, 1983.

Barnes, R. T. H., R. Hide, A. A. White, and C. A. Wilson, Atmospheric Angular Momentum Fluctuations, Length of Day Changes and Polar Motion, Proc. R. Soc. Lon., 387, 31-73, 1983.

Barnett, T. P., The Interaction of Multiple Time Scales in the Tropical Climate System, $J$. Climate, 4, 269, 1991.

Beutler, G., and E. Brockman, eds, Proceedings of the 1993 International GPS Service for Geodynamics (IGS) Workshop, Druckerei der Universitat Bern, 1993.

Brosche, P., and J. Wünsch, On the Rotational Angular Momentum of the Oceans and the Corresponding Polar Motion, Astron. Nach., 315, 181-188, 1994.

Brosche, P. U. Seiler, J. Sündermann and J. Wünsch, Periodic Changes in Earth's Rotation due to Oceanic Tides, Astron. Astrophys., 220, 318-320, 1991.

Brosche, P., J. Wünsch, J. Campbell and H. Schuh, Ocean Tide Effects in Universal Time detected by VLBI, Astron., Astrophys., 245, 676-682, 1991.

Capitaine, N., "Corrections to Some Terms of Nutation Deduced from the Paris Astrolabe Observations," in Nutation and the Earth's Rotation, eds. E. P. Federov, M. L. Smith and P. L. Bender, 87-94, 1980.

Cazenave, A. (ed.), Earth Rotation: Solved and Unsolved Problems, NATO Advanced Institute Series C: Mathematical and Physical Sciences Vol. 187, ed. A. Cazenave, D. Reidel, Boston, 1986.

Chao, B. F., Interannual Length of Day Variations with Relation to the Southern Oscillation/El Niño, Geophys. Res. Letts., 11, 541-544, 1984.

Chao, B. F., Correlation of Interannual Lengths-of-Day Variation with El Niño/Southern Oscillation, 1972-1986, J. Geophys. Res., 93, B7, 7709-7715, 1988.

Chao, B. F., Length-of-Day Variations Caused by El Niño/Southern Oscillation and the Quasi-Biennial Oscillation, Science, 243, 923-925, 1989.

Chao, B. F., and A. Y. Au, Atmospheric Excitation of the Earth's Annual Wobble, 19801988, J. Geophys. Res., 96, 6577-6582, 1991.

Chen, W. Y., Assessment of Southern Oscillation Sea-Level Pressure Indices., Mon. Weather Rev., 110, 800-807, 1982.

Cheng, M. K., R. J. Eanes, C. K. Shum, B. E. Schutz, and B. D. Tapley, Temporal Variation in Low Degree Zonal Harmonics from Starlette Orbit Analysis, Geophys. Res. Lett., 16, 393-396, 1989.

Cheng, M. K., R. J. Eanes, B. D. Tapley, Tidal Deceleration of the Moon's Mean Motion, Geophys. J. Intern., 108, 401-409, 1991.

Clark, T. A., J. W. Ryan, and K. D. Baver, ERDE: High Resolution Observations of Earth Orientation Parameters by Very Long Baseline Interferometry, EOS, Trans. Amer. Geophys. Union, 71, 1271, 1990.

Dickey, J. O., Atmospheric Excitation of the Earth's Rotation: Progress and Prospects via Space Geodesy, in Contributions of Space Geodesy to Geodynamics: Earth Dynamics, Geodyn. Ser., vol. 24, edited by D. E. Smith and D. L. Turcotte, pp. 55-70, AGU, Washington, D. C., 1993.

Dickey, J. O., High Time Resolution Measurements of Earth Rotation, Advances in Space Research, 13, 185-196, 1993. 
Dickey, J. O., and T. M. Eubanks, The Application of Space Geodesy to Earth Orientation Studies, Space Geodesy and Geodynamics (eds.) by A. J. Anderson and A. Cazenave, Academic Press, New York, 221-269, 1986.

Dickey, J. O., and M. Feissel, eds., Results from the SEARCH'92 Campaign, International Earth Rotation Service Technical Note 16, Observatoire de Paris, Paris, 1994.

Dickey, J. O., T. M. Eubanks, and R. Hide, Interannual and Decade Fluctuations in the Earth's Rotation, Variations in the Earth's Rotation, Geophysical Monograph Series of the American Geophysical Union, Washington, D. C., D. McCarthy (ed.), 157-162, 1990.

Dickey, J. O., M. Ghil, and S. L. Marcus, Extratropical Aspects of the 40-50 Day Oscillation in Length-of-Day and Atmospheric Angular Momentum, J. Geophys. Res., 96, 22, 643-22, 658, 1991.

Dickey, J. O., S. L. Marcus and R. Hide, Global Propagation of Interannual Fluctuations in Atmospheric Angular Momentum, Nature, 357, 484-488, 1992a.

Dickey, J. O., S. L. Marcus, J. A. Steppe and R. Hide, The Earth's Angular Momentum Budget on Subseasonal Time Scales, Science, 255, 321-324, $1992 \mathrm{~b}$.

Dickey, J. O., S. L. Marcus, T. M. Eubanks, and R. Hide, Climate Studies via Space Geodesy: Relationships Between ENSO and Interannual Length-of-Day Variations, in Interactions Between Global Climate Subsystems: The Legacy of Hann, Geophys. Monogr. Ser., vol. 75, edited by G. A. McBean and M. Hantel, pp. 141-155, AGU, Washington, D. C., 1993a.

Dickey, J. O., S. L. Marcus, C. M. Johns, R. Hide and S. R. Thompson, The Oceanic Contribution to the Earth's Seasonal Angular Momentum Budget, Geophys. Res. Lett., 20, 2953-2956, 1993 b.

Dickey, J. O., P. L. Bender, J. E. Faller, X X Newhall, R. L. Ricklefs, J. G. Ries, P. J. Shelus, C. Veillet, A. L. Whipple, J. R. Wiant, J. G. Williams, and C. F. Yoder, Lunar Laser Ranging: A Continuing Legacy of the Apollo Program, Science, 265, 482-490, 1994a.

Dickey, J. O., S. L. Marcus, R. Hide, T. M. Eubanks, and D. H. Boggs, Angular Momentum Exchange Among the Solid Earth, Atmosphere, and Oceans: A Case Study of the 1982-83 El Niño Event, J. Geophys. Res., 99, B12, 23, 921-23, 937, 1994b.

Dickey, J. O., T. A. Clark, T. M. Eubanks, M. Feissel, W. G. Melbourne, J. R. Ray, D. A. Salstein, B. E. Schutz, and C. Veillet, SEARCH'92 Campaign, An Overview, International Earth Rotation Service Technical Note 16, Observatoire de Paris, Paris, 1994c.

Dickman, S. R., Ocean Tides for Satellite Geodesy, Mar. Geod., 14, 21-56, 1991.

Dickman, S. R., Dynamic Ocean Tide Effects on Earth's Rotation, Geophys. J. Int. , 112 , 448-470, 1993.

Eubanks, T. M., Variations in the Orientation of the Earth, in Contributions of Space Geodesy to Geodynamics: Earth Dynamics, Geodyn. Ser., vol. 24, edited by D. E. Smith and D. L. Turcotte, pp. 1-54, AGU, Washington, D. C., 1993.

Eubanks, T. M., J. A. Steppe, J. O. Dickey, R. D. Rosen and D. A. Salstein, 1988: Causes of rapid motions of the Earth's pole. Nature, 334, 115-119, 1988.

Eubanks, T. M., J. O. Dickey, and J. A. Steppe, The 1982-83 El Niño, the Southern Oscillation, and Changes in the Length of Day, Trop. Ocean and Atmos. Newsletter, 29, 21-23, 1985a.

Eubanks, T. M., J. A. Steppe, J. O. Dickey, and P. S. Callahan, A Spectral Analysis of the Earth's Angular Momentum Budget, J. Geophys. Res., 90, B7, 5385-5404, 1985 b. 
Eubanks, T. M., J. A. Steppe, and J. O. Dickey, The El Niño, the Southern Oscillation and the Earth's Rotation, in Earth Rotation; Solved and Unsolved Problems, NATO Advanced Institute Series C: Mathematical and Physical Sciences, 187, ed. A. Cazenave, 163-186, D. Reidel, Norwell, Mass., 1986.

Feissel, M., Determination of the Earth Rotation Parameters by the BIH 1962-1979, Bull. Geod., 54, 81-102, 1980.

Feissel, M. and D. Gambis, La Mise en Evidence de Variations Rapides de la Durée du Jour, C. R. Acad. Sci. Paris, Series B, 291, 271-273, 1980.

Feissel, M. and C. Nitschelm, Time Dependent Aspects of the Atmospheric Driven Fluctuations in the Duration of the Day, Ann. Geophys., 3, 180-186, 1985.

Feissel, M., D. Bourquard, P. Charlot, E. Eisop, N. Essaifi, D. Gambis, J.-F. Lestrade, E. F. Arias, C. Boucher, Z. Altamimi, Earth Orientation and Related Reference Frames, Space Geodesy and Geodynamics, Geophysical Monograph Series of the American Geophysical Union, Washington, D. C., D. L. Turcotte (ed.), 99-112, 1993.

Freedman, A. P., J. A. Steppe, J. O. Dickey, T. M. Eubanks, and L. Y. Sung, The ShortTerm Prediction of Universal Time and Length of Day Using Atmospheric Angular Momentum, J. Geophys. Res., 99, 6981-1996, 1994a.

Freedman, A. P., R. Ibañez-Meier, T. A. Herring, S. M. Lichten, and J. O. Dickey, Subdaily Earth Rotation During the Epoch ‘92 Campaign, Geophys. Res. Lett., 21, 769772, 1994b.

Fricke, W., Arguments in Favor of a Change in Precession, Astron. Astrophys., 54, 363$366,1977$.

Fricke, W., Definition and Practical Realization of the Reference Frame in the FK5-the Role of Planetary Dynamics and Stellar Kinematics in the Definition, in Reference Coordinate Systems for Earth Dynamics, F, M. Gaposchkin and B. Kolaczek (eds.), D. Reidel, 331-340, 1981.

Ghil, M., Dynamics, Statistics and Predictability of Planetary Flow Regimes, In Irreversible Phenomena and Dynamical Systems Analysis in Geosciences, C. and G. Nicolis (eds.), Reidel, Dordrecht/Boston, 241-283, 1987.

Ghil, M., and S. Childress, Topics in Geophysical Fluid Dynamics: Atmospheric Dynamics, Dynamo Theory and Climate Dynamics, Springer-Verlag, New York/Berlin, 485, 1987.

Gill, A. E., Atmosphere Ocean Dynamics, Academic, New York, 1982.

Gross, R. S., The Influence of Earthquakes on the Chandler Wobble During 1973-1983, Geophys. J. R. Astr. Soc., 85, 161-177, 1986.

Gross, R. S., The Secular Drift of the Rotation Pole, Earth Rotation and Coordinate Reference Frames, C. Boucher and G. A. Wilkins (eds.), Springer-Verlag, New York, 146-153, 1990.

Gross, R. S., The Effect of Ocean Tides on the Earth's Rotation as Predicted by the Results of an Ocean Tide Model, Geophys. Res. Lett., 20, 293-296, 1993.

Gross, R. S., A Combination of Earth Orientation Data: SPACE93, in IERS Technical Note 17: Earth Orientation, Reference Frame and Atmospheric Excitation Functions, P. Charlot (ed), Observatoire de Paris, Paris, France, C5-C12, 1994.

Gross, R. S., and U. J. Lindqwister, Atmospheric Excitation of Polar Motion during the GIG'91 Measurement Campaign, Geophys. Res. Lett., 19, 849-852, 1992.

Guinot, B., Rotation of the Earth and Polar Motion Services In Proc. GEOP Conf. Int. Symp. Appl. Geod. Geodyn. 9th, pp. 13-18, Dept. of Geodetic Science, Rep. No. 280, Ohio State Univ., 1978. 
Gwinn, C. R., T. A. Herring, and I. I. Shapiro, Geodesy by Radio Interferometry: Studies of the Forced Nutations of the Earth 2 Interpretation, J. Geophys. Res., 91, 4755-4765, 1986.

Herring, T. A., and D. Dong, Current and Future Accuracy of Earth Orientation Measurements, Proceedings of the AGU Chapman Conference on Geodetic VLBI: Monitoring Global Change (NOAA Technical Report NOS 137 NGS 49), 306-324, 1991.

Herring, T. A., and D. Dong, Measurement of Diurnal and Semidiurnal Rotation Variations and Tidal Parameters of the Earth, J. Geophys. Res., 99, 18,051-18,071, 1994.

Herring, T. A., C. R. Gwinn, and I. I. Shapiro, Geodesy by Radio Interferometry: Studies of the Forced Nutations of the Earth 1. Data Analysis, J. Geophys. Res., 91, 4745-4754, 1986.

Herring, T. A., B. A. Buffett, P. M. Mathews, and I. I. Shapiro, Forced Motions of the Earth: Influence of Inner Core Dynamics: 3. Very Long Baseline Interferometry Data Analysis, J. Geophys. Res., 96, 8259-8273, 1991.

Hide, R., Interaction Between the Earth's Liquid Core and Solid Mantle, Nature, 222, 1055-1056, 1969.

Hide, R., Towards a Theory of Irregular Variations in the Length of the Day and CoreMantle Coupling, Phil. Trans. Roy. Soc., A284, 547-554, 1977.

Hide, R. Fluctuations in the Earth's Rotation and the Topography of the Core-Mantle Interface, Phil. Trans. Roy. Soc., A328, 351-363, 1989.

Hide, R., and J. O. Dickey, Earth's Variable Rotation, Science, 253, 629, 1991.

Hide, R., N. T. Birch, L. V. Morrison, D. J. Shea, and A. A. White, Atmospheric Angular Momentum Fluctuations and Changes in the Length of the Day, Nature, 286, 114-117, 1980.

Hide, R., R. W. Clayton, B. H. Hager, M. A. Spieth, and C. V. Voorhies, Topographic Core-Mantle Coupling and Fluctuations in the Earth's Rotation, Geophysical Monograph Series of the American Geophysical Union, Washington D. C., K.-I. Aki (ed), 107-120, 1993.

Horel, J. D., and J. M. Wallace, Planetary-Scale Atmospheric Phenomena Associated with the Southern Oscillation, Mon. Weather Rev., 109, 813-828, 1981.

Jackson, A., J. Bloxham, and D. Gubbins, Time-Dependent Flow at the Core Surface and Conservation of Angular Momentum in the Coupled Core-Mantle System, Dynamics of Earth's Deep Interior and Earth Rotation, Geophysical Monograph 72, IUGG Volume 12, American Geophysical Union, 1993.

Jault, D., andJ-L. Le Mouël, The Topographic Torque Associated with a Tangentially Geostrophic Motion at the Core Surface and Inferences on the Flow Inside the Core, Geophys. Astrophys. Fluid. Dynam., 48, 273-296, 1989.

Jault, D., and J-L. Le Mouël, Exchange of Angular Momentum Between the Core and Mantle, J. Geomag. Geoelect., 43, 111-129, 1991.

Jordi, C., L. V. Morrison, R. D. Rosen, D. A. Salstein, and G. Rossello, Fluctuations in the Earth's Rotation Since 1830 from High Resolution Astronomical Data, Geophys. J. Intern., 117, 811-818, 1994.

Keppenne, C. L., and M. Ghil, Adaptive Spectral Analysis of the Southern Oscillation Index, in Proceedings of the XVth Annual Climate Diagnostics Workshop, 30-35, U. S. Department of Commerce, NOAA, 1991.

Kinoshita, H., Theory of the Rotation of the Rigid Earth, Celest. Mech., 15, 277-326, 1977. 
Krishnamurti, T.N. and D. Subrahmanyam, The 30-50 Day Mode at $850 \mathrm{mb}$ During MONEX, J. Atmos. Sci., 39, 2088-2095, 1982.

Kuehne, J. W., and C. R. Wilson, Terrestrial Water Storage and Polar Motion, J. Geophys. Res., 96, B3, 4337-4345, 1991.

Lambeck, K., The Earth's Variable Rotation, Cambridge Univ. Press, London and New York, 1980a.

Lambeck, K., Changes in Length of Day and Atmospheric Circulation, Nature, 26, 104, 1980b.

Lambeck, K., Geophysical Geodesy, The Slow Deformation of the Earth, Clarendon Press, Oxford, 1988.

Lambeck, K., and A. Cazenave, Philos. Trans. R. Soc. London A 284, 495-506, 1977.

Langley, R. B., R. W. King, I. I. Shapiro, R. D. Rosen, and D. A. Salstein, Atmospheric Angular Momentum and the Length of the Day: A Common Fluctuation with a Period Near 50 Days, Nature, 294, 730-733, 1981.

Madden, R. A., Seasonal Variations of the 40-50 Day Oscillation in the Extratropics, J. Atmos. Sci., 43, 3138-3158, 1986.

Madden, R. A., Relationships Between Changes in the Length of Day and the 40- to 50Day Oscillation in the Tropics, J. Geophys. Res., 92, 8391-8399, 1987.

Madden, R. A. and P. R. Julian, Detection of a 40-50 Day Oscillation in the Zonal Wind in the Tropical Pacific, J. Atmos. Sci., 28, 702-708, 1971.

Madden, R. A. and P. R. Julian, Description of Global-Scale Circulation Cells in the Tropics with a 40-50 Day Period, J. Atmos. Sci., 29, 1109-1123, 1972.

Marcus, S. L., Intraseasonal Oscillations in the Earth-Atmosphere System, Ph.D. Thesis, University of California at Los Angeles, 1990.

Marcus, S. L., and J. O. Dickey, Coupled Poleward Propagation of Sea Surface Temperature and Atmospheric Angular Momentum Anomalies: Results from AMIP, Sixth Conference on Climate Variations, American Meteorological Society, 70-74, 1994.

Marcus, S. L., M. Ghil, J. O. Dickey, and T. M. Eubanks, Origin of the 30-60 Day Oscillation in the Length of Day and Atmospheric Angular Momentum: New Findings from the UCLA General Circulation Model, in The Earth's Rotation and Reference Frames for Geodesy and Geodynamics, G. A. Wilkins (ed.), Springer-Verlag, New York, 98-105, 1990.

Marsh, J. G., et al., GEM-T2 Gravitation Model, J. Geophys. Res., 95, 22,043-22,047, 1990.

Mathews, P. M., and I. I. Shapiro, Nutations of the Earth, Annu. Rev. Earth Planet. Sci., 20, 469-500, 1992.

Mathews, P. M., B. A. Buffet, T. A. Herring, and I. I. Shapiro, Forced Nutations of the Earth: Influence of Inner Core Dynamics 2. Numerical Results and Comparisons, $J$. Geophys. Res., 96, 8243-57, 1991.

McCarthy, D. D., and A. K. Babcock, The Length of Day Since 1656, Phys. Earth and Planet. Inter., 44, 281-292, 1986.

Merriam, J. B., Meteorological Excitation of the Annual Polar Motion, Geophys. J. R. Astron. Soc., 70, 41-56, 1982.

Miller, A. J., Periodic Variation of Atmospheric Circulation at 14-16 Days, J. Atmos. Sci., 31, 720-726, 1974.

Morgan, P. J., R. W. King, and I. I. Shapiro, Length of Day and Atmospheric Angular Momentum: A Comparison for 1981-1983, J. Geophys. Res., 90, 12645-12652, 1985. 
Moritz, H., and I. I. Mueller, Earth Rotation: Theory and Observation, The Ungar Publishing Co., New York, 1987.

Munk, W. H., and G. J. F. MacDonald, The Rotation of the Earth, Cambridge University Press, 1960.

Murakami, M., Large-scale Aspects of Deep Convective Activity Over the GATE Area, Mon. Weather Rev., 107, 994-1013, 1979.

Nakiboglu, S. M. \& K. Lambeck, Geophys. J. R. Astr. Soc., 62, 49-58, 1980.

Neuberg, J., J. Hinderer, and W. Zurn, Stacking Gravity-Tied Observations in Central Europe for the Retrieval of the Complex Eigenfrequency of the Nearly Diurnal FreeWobble, Geophys. J. R. Ast. Soc., 91, 853-868, 1987.

Paulus, J., and M. Stix, Electromagnetic Core-Mantle Coupling, Earth Rotation: Solved and Unsolved Problems, A. Cazenave, ed., D. Reidel Publishing Co., Dordrecht, 259267, 1986.

Philander, S. G. H., El Niño Southern Oscillation Phenomena, Nature, 302, 295-301, 1983.

Philander, S. G. H., El Niño, La Niña, and the Southern Oscillation, Academic Press, New York, 1990.

Ponte, R. M., Barotropic Motions and the Exchange of Angular Momentum Between the Oceans and Solid Earth, J. Geophys. Res., 95, 11,369-11,374, 1990.

Ponte, R. M. and R. D. Rosen, Oceanic Angular Momentum and Torques in a GeneralCirculation Model, J. Phys. Ocean., 24, 1966-1977, 1994.

Rasmusson, E. U., and J. M. Wallace, Meteorological Aspects of the El Niño/Southern Oscillation, Science, 222, 1195-1202, 1983.

Rasmusson, E. M., X. Wang, and C. F. Ropelewski, The Biennial Component of ENSO Variability, J. Mar. Systems, 1, 71-96, 1990.

Rochester, M. G., Causes of Fluctuations in the Earth's Rotation, Philos. Trans. R. Soc. London A, 313, 95-105, 1984.

Rosen, R. D., The Axial Momentum Balance of Earth and Its Fluid Envelope, Surveys Geophys., 14, 1-29, 1993.

Rosen, R. D., and D. A. Salstein, Variations in Atmospheric Angular Momentum on Global and Regional Scales and the Length of Day, J. Geophys. Res., 88, C9, 5451$5470,1983$.

Rosen, R. D., and D. A. Salstein, Contribution of Stratospheric Winds to Annual and Semi-Annual Fluctuations in Atmospheric Angular Momentum and the Length of Day, J. Geophys. Res., 90, 8033-8041, 1985.

Rosen, R. D., and D. A. Salstein, Comment on "A Seasonal Budget of Earth's Axial Angular Momentum" by Naito and Kikucho, Geophys. Res. Lett., 18, 1925-1926, 1991.

Rosen, R. D., D. A. Salstein, T. M. Eubanks, J. O. Dickey, and J. A. Steppe, An El Niño Signal in Atmospheric Angular Momentum and Earth Rotation, Science, 225, 411-414, 1984.

Rosen, R. D., K. Arpe, A. J. Miller, and D. A. Salstein, Accuracy of Atmospheric Angular Momentum Estimates from Operational Analyses, Monthly Weather Rev., 115, 1627. 1639, 1987.

Rosen, R. D., D. A. Salstein, T. M. Wood, Discrepancies in the Earth-Atmosphere Angular Momentum Budget, Journal of Geophysical Research, 95, 265-279, 1990.

Salstein, D. A., Monitoring Atmospheric Winds and Pressures for Earth Orientation Studies, Adv. Space R., 13, 175-184, 1993.

Salstein, D. A., and R. D. Rosen, Earth Rotation as a Proxy for Interannual Variability in Atmospheric Circulation, 1860-Present, J. Clim. and Appl. Meteorol., 25, 1870-1877, 1986. 
Salstein, D. A., and R. D. Rosen, Topographic Forcing of the Atmosphere and a Rapid Change in the Length of Day, Science, 264, 407-409, 1994.

Salstein, D. A., D. M. Kann, A. J. Miller, and R. D. Rosen, The Sub-Bureau for Atmospheric Angular Momentum of the International Earth Rotation Service: A Meteorological Data Center with Geodetic Applications, Bull. Am. Meteorol. Soc., 74, 67-80, 1993.

Schwiderski, E., Atlas of Ocean Tidal Charts and Maps, Part I: The Semidiurnal Principal Lunar Tide M2, Mar. Geod., 6, 219-256, 1983.

Seidelmann, P. K., 1980 IAU Nutation: The Final Report of the IAU Working Group on Nutation, Celest. Mech., 27, 79-106, 1982.

Seiler, U., Periodic Changes of the Angular Momentum Budget Due to the Tides of the World Ocean, J. Geophys. Res., 96, 10287-10300, 1991.

Simmons, A. J., J. M. Wallace, and G. W. Branstator, Barotropic Wave Propagation and Instability and Atmospheric Teleconnection Patterns, 40, 1363-1392, 1983.

Smith, M. L., and F. A. Dahlen, The Period and $Q$ of the Chandler Wobble, Geophys. J. $R$. Astron. Soc., 64, 223-281, 1981

Sovers, O. J., C. S. Jacobs, and R. S Gross, Measuring Rapid Ocean Tidal Earth Orientation Variations with VLBI, J. Geophys. Res., 98, 19,959-19,971, 1993.

Stephanick, M., Interannual Atmospheric Angular Momentum Variability 1963-1973 and the Southern Oscillation, J. Geophys. Res., 87, 428-432, 1982.

Stephenson, F. R., and L. V. Morrison, Long-Term Changes in the Rotation of the Earth: 700 B.C. to A.D. 1980, Phil. Trans. R. Soc. London, A313, 47-70, 1984.

Stephenson, F. R., and L. V. Morrison, Long-Term Fluctuation in the Earth's Rotation: 700 B.C. to A.D. 1990, Phil. Trans. R. Soc. London, Series A, in press, 1995.

Wahr, J. M., The Forced Nutations of an Elliptical, Rotating, Elastic, and Oceanless Earth, Geophys. J. Roy. Astron. Soc., 64, 705-727, 1981.

Wahr, J. M., Friction and Mountain Torque Estimates from Global Atmospheric Data, $J$. Atmos. Sci. 41, 190, 1984.

Wahr, J. M., The Earth's Rotation, Ann. Rev. Earth Planet Sci., 16, 231-249, 1988.

Wahr, J., H. Dazhong, A. Trupin and J. Lindquist, Secular Changes in Rotation and Gravity: Evidence of Post-Glacial Rebound or of Changes in Polar Ice?, Adv. Space Res., 13, 128-136, 1993.

Watkins, M., M., R. J. Eanes, Diurnal and Semidiurnal Variations in Earth Orientation Determined from LAGEOS Laser Ranging, J. Geophys. Res., 99, 9, 18,073-18,079, 1994.

Williams, J. G., X X Newhall, J. O. Dickey, Luni-solar Precession: Determination from Lunar Laser Ranging, Astron. Astrophys. Lett., 241, L9-L12, 1991.

Wilson, C. R., Contribution of Water Mass Redistribution to Polar Motion Excitation in Contributions of Space Geodesy to Geodynamics: Earth Dynamics, Geodynamics Series vol. 24, eds. D. E. Smith and D. L. Turcotte, American Geophysical Union, D. C., 77-82, 1993.

Wilson, C. R., and R. O. Vincente, An Analysis of the Homogeneous ILS Polar Motion Series, Geophys. J. R. Astron. Soc., 62, 605-616, 1980.

Wolf, W. L., and R. B. Smith, Length-of-Day Changes and Mountain Torque During El Niño, J. Atmos. Sci., 44, 3656-3660, 1987.

Wyrtki, K., The Slope of Sea Level Along the Equator During the 1982/1983 El Niño, J. Geophys. Res., 89, 10,419-10,424, 1984.

Wu, P., and W. Peltier, Pleistocene Deglaciation and the Earth's Rotation: A New Analysis, Geophys. J. R. Astr. Soc., 76, 753-791, 1984. 
Wünsch, J, and U. Seiler, Theoretical Amplitudes and Phases of the Periodic Polar Motion Terms Caused by Ocean Tides, Astron. Astrophys., 266, 581-587, 1992.

Wyrtki, K., Water Displacements in the Pacific and the Genesis of El Niño Cycles, $J$. Geophys. Res., 90, 7129-7132, 1985.

$\mathrm{Xu}$, J. S., On the Relationship Between the Stratospheric Quasi-Biennial Oscillation and the Tropospheric Southern Oscillation, J. Atmos. Sci., 49, 725-734, 1992.

Yasunari, T., Structure of an Indian Summer Monsoon System With Around 40-day Period, J. Meteor. Soc. Japan, 59, 336-354, 1981.

Yoder, C. F., M. W. Parke, and J. G. Williams, Tidal Variations of the Earth's Rotation, J. Geophys. Res., 86, B2, 881-891, 1981.

Yoder, C. F., J. G. Williams, J. O. Dickey, B. E. Schutz, R. J. Eanes, and B. D. Tapley, Secular Variation of Earth's Gravitational Harmonic $\mathrm{J}_{2}$ Coefficient from Lageos and Nontidal Acceleration of Earth Rotation, Nature, 303, 757-762, 1983.

Yumi, S. and K. Yokoyama, Results of the International Latitude Service in a Homogeneous System 1899.9-1979.0, Publications, Central Bureau of the International Polar Motion Service, Mizusawa, 1980.

Zheng, D., Chin. Astron. Astrophys. 3, 114, 1979. 